Research Article

\title{
The Association between SLC25A15 Gene Polymorphisms and Hyperornithinemia-hyperammonemia-homocitrullinuria Syndrome: Using In Silico Analysis
}

\author{
Nuha A. Mahmoud ${ }^{1 *}$, Dina T. Ahmed ${ }^{2 *}$, Zainab O. Mohammed ${ }^{3}$, Fatima A. Altyeb ${ }^{2}$, \\ Mujahed I. Mustafa ${ }^{2}$, Mohamed A. Hassan ${ }^{2,4}$
}

1- Department of Biochemistry, Faculty of Medicine, National University, Khartoum, Sudan.

2- Department of Biotechnology, Africa City of Technology, Khartoum, Sudan.

3- Department of Hematology, Ribat University Hospital, Khartoum, Sudan.

4- Department of Bioinformatics, DETAGEN Genetics Diagnostic Center, Kayseri, Turkey.

*Both authors contributed equally to this manuscript.

Corresponding Author: Nuha A. Mahmoud: nuhaamin@gmail.com

Background: Hyperornithinemia-hyperammonemia-homocitrullinuria $(\mathrm{HHH})$ syndrome is an autosomal recessive inborn error of the urea cycle. It is caused by mutations in the SLC25A15 gene that codes the mitochondrial ornithine transporter. The aim of this study is to detect and identify the pathogenic SNPs in SLC25A15 gene through a combination set of bioinformatics tools and their effect on the structure and function of the protein.

Methods: The deleterious SNPs in SLC25A15 are detected by various bioinformatics tools, with addition to identifying their effects on the structure and function of this gene.

Results: 20 deleterious SNPs out 287of were found to have their own damaging effects on the structure and function of the SLC25A15 gene.

Conclusion: This study is the first in silico analysis of SLC25A15 using a selection of bioinformatics tools to detect functional and structural effects of deleterious SNPs. Finding the pathogenic SNPs is a promising start to innovate new, useful SNP diagnostic markers for medical testing and for safer novel therapies specifically targeting mutant SLC25A15.

\section{Keywords:}

Hyperornithinemia-hyperammonemia-homocitrullinuria (HHH), SLC25A15, SNPs, urea cycle, in silico analysis. 


\section{Introduction:}

Hyperornithinemia-hyperammonemia-homocitrullinuria $(\mathrm{HHH})$ syndrome is an autosomal recessive inborn error of the urea cycle (1-5), (OMIM\#238970) (6-8), it is located on chromosome 13q14(7, 9). This syndrome occurs in the early infancy or childhood period, although, few cases of adult onset have also been reported $(7,10-14)$. HHH syndrome is caused by mutations in the SLC25A15 gene that codes the mitochondrial ornithine transporter, which transports ornithine across the inner mitochondrial membrane from the cytosol to the mitochondrial matrix (15). This is a vital step in the urea cycle, which serves to eliminate toxic ammonium ions from the breakdown of nitrogen $(6,16-21)$. The HHH clinical symptoms are highly variable, which include spastic paraplegia, extrapyramidal signs, stroke-like episodes, hypotonia, seizures, ataxia, protein intolerance, failure to thrive, pyramidal dysfunction developmental delay/intellectual disability, hepatic failure $(13,16,20,22-24)$, and even death can arise if the condition is not diagnosed early and treated quickly (25).

Even though, this disease targets a wide variation of different ethnicities, Canada (23\%), Italy $(17 \%)$ and Japan (13\%) were reported with the highest prevalence rates $(15,26-28)$

Albeit HHH treatment includes a strict treatment protocol consisting control of dietary protein, nitrogen forager medications, as well as, amino acid and vitamins supplementations, still there is no effective therapy for this type of inborn disease $(4,22-25)$, thus identifying the SLC25A15 gene holds a promising future tool to improve both survival rates as well as the quality of life in surviving of $\mathrm{HHH}$ patients $(29,30)$.

Detection and identification of pathogenic SNPs of SLC25A15 through different in silico prediction software are the main aims of this study. As well as, to determine the structure, bio functions and the regulatory mechanism of their respective proteins. This study is the first in silico analysis of the SLC25A15 coding region. Using this approach to identify the pathogenic SNPs with no cost; novel therapies specifically targeting mutant SLC25A15 will open new methods of treatment for $\mathrm{HHH}$ patients(31).

\section{Methods:}

\subsection{Data Mining:}

The SLC25A15 human gene data was obtained from the National Center for Biotechnology Information (NCBI) (http://www.ncbi.nlm.nih.gov/ ). The variant's information of the gene was collected from the NCBI dbSNP database (http://www.ncbi.nlm.nih.gov/snp/ ) and the SLC25A15 protein sequence was obtained from UniProt database (https://www.uniprot.org/).

\subsection{SIFT:}

The Sorting Intolerant from Tolerant (SIFT) algorithm is a standardized tool for detecting all missense SNPs in the protein sequence by searching for homologous sequences and carry out multiple sequence alignment. The score of each variant ranges from 0 to 1 , deleterious SNP is considered when the score is $\leq 0.05$, while tolerated SNP's score is $>0.05(32,33)$ SIFT is an available online tool (34) (http://sift-dna.org). 


\subsection{PolyPhen-2:}

PolyPhen-2 (polymorphism phenotyping v2) is a tool that predicts the impact of substitution of the amino acid on the structure and function of the protein. The input data needs accession number, position of variants, original and mutant amino acids. The PolyPhen figures the PSIC (position-specific independent count) and counts the score difference between the two variants. When resulted with a score of (0.96-1) it's considered 'Probably damaging', while (0.71-0.95) scores considered as 'Possibly damaging and Benign' (0.31-0.7) $(32,35,36)$. PolyPhen-2 is available at (http://genetics.bwh.harvard.edu/pph2/).

\subsection{PROVEAN:}

PROVEAN (Protein Variation Effect Analyzer), is an algorithm, which accurately predicts the impact of single amino acid substitutions, multiple substitutions, insertions and deletions(37). The tool accepts a protein sequence and amino acid variations as input, performs a BLAST to identify homologous sequences, and generates the scores. A variant is predicted to be 'deleterious' if the final score is below -2.5 , and is predicted to be 'neutral' otherwise $(37,38)$. It is available at (http://provean.jcvi.org/index.php).

\subsection{PHD-SNP:}

Predictor of Human Deleterious Single Nucleotide Polymorphisms; is an online Support Vector Machine SVM-based classifier. It predicts whether the amino acid substitution is deleterious or neutral (39). It is available at (http://snps.biofold.org/phd-snp/phd-snp.html ).

\subsection{SNPs\&GO:}

Single Nucleotide Polymorphism Database \& Gene Ontology is a support vector machine (SVM) based method. The information derived from the protein sequence, structure, and protein function; it needs a whole protein in FASTA sequence, and/or its three-dimensional structure, a target SNP and its functional Gene Ontology (GO) terms to be an input option. The output provides, for each protein variation, the probabilities to be disease or neutral. The score of predicted variants is $82 \%$ accurate with Matthews's correlation coefficient of 0.63 and if it is > 0.5 this is defined as a 'disease' (39-41). It is freely available at (http://snps.biofold.org/snps-andgo/snps-and-go.html )

\subsection{PANTHER:}

Protein Analysis through evolutionary relationships, is used for analysis of variants, it utilizes hidden Markov model (HMM) based statistical methods and multiple sequence alignments which create the substitution position-specific evolutionary conservation score (subPSEC). The SNPs is considered deleterious with score $(<-3)$ and neutral with (score $>-3)$ (39). It is available at (http://www.pantherdb.org/)

\subsection{P-Mut:}

P-Mut is an online tool annotates and predicts the damaging mutations. Pmut is rely on sequence information of any protein to identify the mutations. It delivers a very simple output: a yes/no answer and a reliability index. Its methods performance is $84 \%$ overall success rate, and $67 \%$ improvement over random (42-44). It is available at (http://mmb.irbbarcelona.org/PMut/ ). 


\subsection{I-Mutant 3.0:}

I-Mutant is a neural-network-based tool for prediction of protein stability and modulation upon single amino acid substitution. The protein sequence and variant position is needed for input data. The output is the predicted free energy change (DDG), which classifies the prediction into: 'large decrease' (DDG $<-0.5 \mathrm{kcal} / \mathrm{mol}$ ), 'large increase' (DDG $>0.5 \mathrm{kcal} / \mathrm{mol})$, or 'neutral' $(-0.5<$ DDG $<0.5 \mathrm{kcal} / \mathrm{mol})(39,44)$. It is available at (http://gpcr2.biocomp.unibo.it/cgi/predictors/IMutant3.0/I-Mutant3.0.cgi ).

\subsection{MUpro:}

A support vector machine-based bioinformatics tool, MUpro, is used for predicting the stability changes of a protein upon nonsynonymous SNPs. The value of the energy change is assumed, and a confidence score among -1 and 1 for measuring the confidence of the assumption is calculated. A score $<0$ shows that the variable reduces the protein stabilization and vice versa (45). It is available at (http://mupro.proteomics.ics.uci.edu/ ).

\subsection{Project HOPE}

Have Our Protein Explained, is an online next-generation tool used for mutant analysis. HOPE's layout demonstrates the molecular origin of disease's phenotype which results from protein mutation. The Web services and DAS servers are used to collect the data. The sources from which HOPE gathers the data are 'Protein's 3D structure' and the 'UniProt database', of wellannotated protein sequences. For each protein, this data is stored in a PostgreSQL-based information system. The data is processed and used to predict the mutation impact on the 3D structure and the function of the protein. The report is generated, is supported with figures and clarify the effects of mutation. Lastly the report can be utilized to design follow-up experiments, better diagnostic \& medicines (46). Available at (http://www.cmbi.ru.nl/hope/input/).

\subsection{RaptorX}

Since the human SLC25A15 protein's 3D structure is unavailable in the Protein Data Bank, RaptorX software was used to obtain the 3D structural model for wild-type of SLC25A15. RaptorX is a bioinformatics web server predicts the structural property of a protein sequence without the need of any templates. It surpasses other servers, chiefly for proteins without close homologs in PDB or with very sparse sequence profile (47). It is available at (http://raptorx.uchicago.edu/ ).

\subsection{UCSF Chimera:}

This desktop application is for the interactive visualization and analysis of molecular structures and related data. The application features are expanded due to incorporation of the web access with desktop tool. The features include the accomplishing computationally intense work and is able to connect to databases. Chimera is subdivided into a 'Core', which is responsible for basic services and visualization, and 'Extensions', that contain new features with higher functionality.

These extensions are Multialign Viewer, docking, trajectories, visualize large-scale molecular assemblies, etc. chimera images with high resolution. This application can generate an animation (48). Chimera is available at (http://www.cgl.ucsf.edu/chimera/). 


\subsection{GeneMANIA:}

GeneMANIA is an online server generating proposition about gene function, investigating gene lists and prioritizing genes for functional assays. It expands the query list with functionally similar genes that recognizes by using available genomics and proteomics data. The high accuracy of the GeneMANIA prediction algorithm and large database make the GeneMANIA an useful tool for any biologist $(49,50)$. It is available at (http://genemania.org/ ).

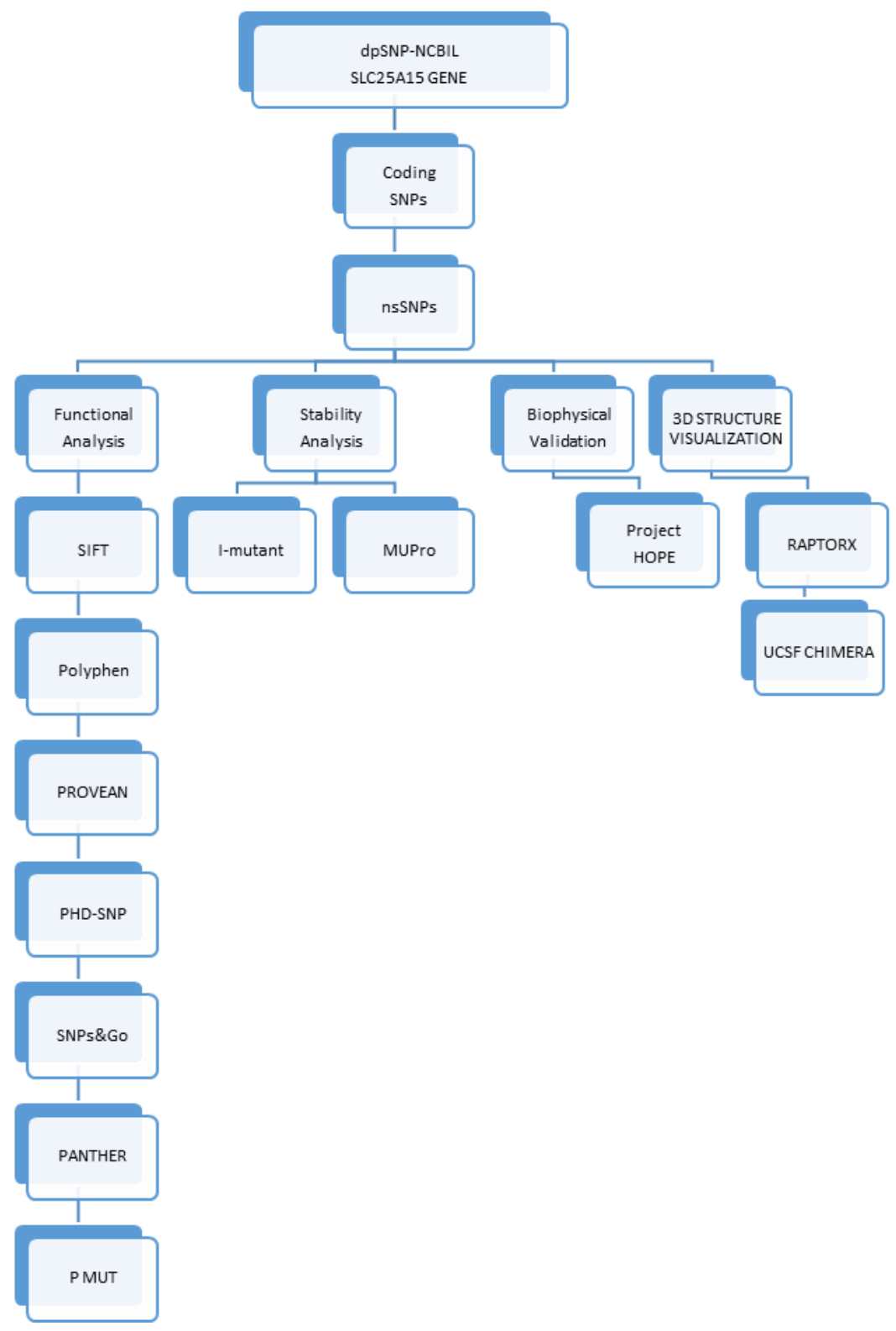

Figure 1: Diagrammatic representation of $S L C 25 A 15$ gene in silico work flow. 


\section{Result and discussion:}

\subsection{Result:}

Table (1): Deleterious - damaging (Affected) nsSNPs predicted by various software:

\begin{tabular}{|c|c|c|c|c|c|c|}
\hline \multirow{2}{*}{$\begin{array}{l}\text { Amino } \\
\text { acid } \\
\text { change }\end{array}$} & \multicolumn{2}{|c|}{ SIFT } & \multicolumn{2}{|c|}{ POLYPHEN-2 } & \multicolumn{2}{|c|}{ PROVEAN } \\
\hline & Prediction & Score & Prediction & Score & $\begin{array}{c}\text { Prediction }(\text { cutoff }= \\
-2.5)\end{array}$ & Score \\
\hline D31H & AFFECTED & 0.00 & probably damaging & 1 & Deleterious & -6.651 \\
\hline D31Y & AFFECTED & 0.00 & probably damaging & 1 & Deleterious & -8.551 \\
\hline Y64C & AFFECTED & 0.00 & probably damaging & 1 & Deleterious & -8.418 \\
\hline G66S & AFFECTED & 0.00 & probably damaging & 1 & Deleterious & -5.634 \\
\hline L71Q & AFFECTED & 0.00 & probably damaging & 1 & Deleterious & -4.892 \\
\hline G86C & AFFECTED & 0.00 & probably damaging & 1 & Deleterious & -8.585 \\
\hline G113C & AFFECTED & 0.00 & probably damaging & 1 & Deleterious & -8.512 \\
\hline G168E & AFFECTED & 0.00 & probably damaging & 1 & Deleterious & -6.745 \\
\hline T176A & AFFECTED & 0.00 & probably damaging & 1 & Deleterious & -4.81 \\
\hline F188S & AFFECTED & 0.00 & probably damaging & 1 & Deleterious & -7.651 \\
\hline G216S & AFFECTED & 0.00 & probably damaging & 1 & Deleterious & -5.543 \\
\hline G217R & AFFECTED & 0.00 & probably damaging & 1 & Deleterious & -5.213 \\
\hline G220R & AFFECTED & 0.00 & probably damaging & 1 & Deleterious & -7.39 \\
\hline K234I & AFFECTED & 0.00 & probably damaging & 1 & Deleterious & -7.488 \\
\hline Q238R & AFFECTED & 0.00 & probably damaging & 1 & Deleterious & -3.795 \\
\hline R275Q & AFFECTED & 0.00 & probably damaging & 1 & Deleterious & -3.64 \\
\hline R275L & AFFECTED & 0.00 & probably damaging & 1 & Deleterious & -6.303 \\
\hline Y287H & AFFECTED & 0.00 & probably damaging & 1 & Deleterious & -4.617 \\
\hline
\end{tabular}




\begin{tabular}{lllcccr} 
E288K & AFFECTED & 0.00 & probably damaging & 1 & Deleterious & -3.773 \\
E288G & AFFECTED & 0.00 & probably damaging & 1 & Deleterious & -6.603 \\
\hline
\end{tabular}

Table (2): Disease-associated nsSNPs predicted by various software:

\begin{tabular}{|c|c|c|c|c|c|c|c|c|c|}
\hline \multirow{2}{*}{$\begin{array}{l}\text { Amino } \\
\text { acid } \\
\text { change }\end{array}$} & \multicolumn{3}{|c|}{ SNP\&GO } & \multicolumn{3}{|c|}{ PHD-SNP } & \multicolumn{3}{|c|}{ PANTHER } \\
\hline & Prediction & $\mathbf{R I}$ & Score & Prediction & RI & Score & Prediction & RI & Score \\
\hline D31H & Disease & 4 & 0.719 & Disease & 7 & 0.863 & Disease & 10 & $0.992 !$ \\
\hline D31Y & Disease & 7 & 0.842 & Disease & 9 & 0.933 & Disease & 10 & 0.995 \\
\hline Y64C & Disease & 6 & 0.797 & Disease & 8 & 0.915 & Disease & 8 & 0.905 \\
\hline G66S & Disease & 6 & 0.794 & Disease & 8 & 0.908 & Disease & 10 & 1 \\
\hline L71Q & Disease & 5 & 0.77 & Disease & 6 & 0.793 & Disease & 6 & 0.82 \\
\hline G86C & Disease & 1 & 0.539 & Disease & 4 & 0.721 & Disease & 5 & 0.775 \\
\hline G113C & Disease & 6 & 0.801 & Disease & 8 & 0.89 & Disease & 8 & 0.916 \\
\hline G168E & Disease & 7 & 0.826 & Disease & 7 & 0.828 & Disease & 7 & 0.85 \\
\hline T176A & Disease & 2 & 0.609 & Disease & 1 & 0.574 & Disease & 0 & 0.525 \\
\hline F188S & Disease & 6 & 0.78 & Disease & 8 & 0.877 & Disease & 6 & 0.814 \\
\hline $\mathrm{G} 216 \mathrm{~S}$ & Disease & 4 & 0.718 & Disease & 6 & 0.793 & Disease & 10 & 0.988 \\
\hline G217R & Disease & 6 & 0.779 & Disease & 7 & 0.874 & Disease & 2 & 0.618 \\
\hline G220R & Disease & 7 & 0.841 & Disease & 8 & 0.914 & Disease & 8 & 0.882 \\
\hline K234I & Disease & 6 & 0.799 & Disease & 7 & 0.874 & Disease & 10 & 0.999 \\
\hline Q238R & Disease & 4 & 0.7 & Disease & 6 & 0.799 & Disease & 3 & 0.633 \\
\hline R275Q & Disease & 5 & 0.76 & Disease & 7 & 0.847 & Disease & 10 & 0.986 \\
\hline $\mathrm{R} 275 \mathrm{~L}$ & Disease & 7 & 0.851 & Disease & 9 & 0.932 & Disease & 10 & 0.991 \\
\hline Y287H & Disease & 3 & 0.649 & Disease & 7 & 0.868 & Disease & 3 & 0.65 \\
\hline E288K & Disease & 5 & 0.743 & Disease & 9 & 0.943 & Disease & 0 & 0.503 \\
\hline E288G & Disease & 1 & 0.537 & Disease & 7 & 0.875 & Disease & 1 & 0.558 \\
\hline
\end{tabular}


Table (3): Disease-associated nsSNPs predicted by P-Mut software:

\begin{tabular}{|c|c|c|}
\hline $\begin{array}{l}\text { Amino acid } \\
\text { change }\end{array}$ & P-Mut Prediction & P-Mut Score \\
\hline $\mathrm{D} 31 \mathrm{H}$ & Disease & $0.85(91 \%)$ \\
\hline D31Y & Disease & $0.86(91 \%)$ \\
\hline Y64C & Disease & $0.85(91 \%)$ \\
\hline G66S & Disease & $0.85(91 \%)$ \\
\hline L71Q & Disease & $0.76(88 \%)$ \\
\hline G86C & Disease & $0.76(88 \%)$ \\
\hline G113C & Disease & $0.86(91 \%)$ \\
\hline G168E & Disease & $0.73(87 \%)$ \\
\hline T176A & Disease & $0.71(86 \%)$ \\
\hline F188S & Disease & $0.86(91 \%)$ \\
\hline G216S & Disease & $0.63(83 \%)$ \\
\hline G217R & Disease & $0.86(91 \%)$ \\
\hline G220R & Disease & $0.86(91 \%)$ \\
\hline K234I & Disease & $0.86(91 \%)$ \\
\hline Q238R & Disease & $0.74(87 \%)$ \\
\hline $\mathrm{R} 275 \mathrm{Q}$ & Disease & $0.86(91 \%)$ \\
\hline $\mathrm{R} 275 \mathrm{~L}$ & Disease & $0.86(91 \%)$ \\
\hline Y287H & Disease & $0.80(89 \%)$ \\
\hline E288K & Disease & $0.85(91 \%)$ \\
\hline E288G & Disease & $0.85(91 \%)$ \\
\hline
\end{tabular}


Table (4): The effect of the deleterious SNPs on the stability predicted by 2 software:

\begin{tabular}{|c|c|c|c|c|c|}
\hline \multirow{2}{*}{$\begin{array}{l}\text { Amino } \\
\text { acid } \\
\text { change }\end{array}$} & \multicolumn{3}{|c|}{ I- mutant } & \multicolumn{2}{|c|}{ MUPRO } \\
\hline & $\begin{array}{c}\text { SVM2 Prediction } \\
\text { Effect }\end{array}$ & $\mathbf{R I}$ & $\begin{array}{l}\text { DDG Value } \\
\text { Prediction }\end{array}$ & Prediction & Score \\
\hline D31H & Decrease & -0.16 & 1 & Decrease & -1.1775469 \\
\hline D31Y & Increase & 0.46 & 3 & Decrease & -0.68362433 \\
\hline Y64C & Decrease & -1.33 & 3 & Decrease & 1.09234 \\
\hline G66S & Decrease & -1.5 & 9 & Decrease & -0.66844573 \\
\hline L71Q & Decrease & -2.24 & 9 & Decrease & -1.8046296 \\
\hline G86C & Decrease & 6 & -1.12 & Decrease & -0.46574415 \\
\hline G113C & Decrease & 1 & -0.64 & Decrease & -0.13197126 \\
\hline G168E & Increase & 3 & -0.54 & Decrease & -0.40969468 \\
\hline T176A & Decrease & 8 & -1.18 & Decrease & -1.526078 \\
\hline F188S & Decrease & 7 & -1.62 & Decrease & -1.740386 \\
\hline G216S & Increase & -0.76 & 1 & Decrease & -0.98156517 \\
\hline G217R & Increase & -0.34 & 1 & Decrease & -1.1923443 \\
\hline \multirow{2}{*}{$\begin{array}{l}\text { G220R } \\
\text { K234I }\end{array}$} & Decrease & -0.39 & 1 & Decrease & -0.50470067 \\
\hline & Decrease & 0.19 & 1 & Increase & 0.014638836 \\
\hline Q238R & Decrease & -0.22 & 2 & Decrease & -1.0081246 \\
\hline R275Q & Decrease & -0.93 & 7 & Decrease & -0.6423489 \\
\hline R275L & Decrease & -0.32 & 7 & Decrease & -0.007036716 \\
\hline Y287H & Decrease & -1.37 & 6 & Decrease & -1.6533478 \\
\hline E288K & Decrease & -0.75 & 8 & Decrease & -1.1400911 \\
\hline E288G & Decrease & -1.14 & 7 & Decrease & -1.3861129 \\
\hline
\end{tabular}


Table (5): The SCL25A15 gene function and its appearance in network and genome:

\section{Function}

alpha-amino acid metabolic process

nitrogen cycle metabolic process

urea metabolic process

acute inflammatory response

cellular amino acid catabolic process

amide biosynthetic process

inflammatory response

complement activation

cellular modified amino acid metabolic process

carboxylic acid catabolic process

organic acid catabolic process

protein activation cascade

alpha-amino acid catabolic process

small molecule catabolic process
FDR

$1.90205 \mathrm{E}-06$

0.001517377

0.001517377

0.003303863

0.009215468

0.022215719

0.028672552

0.031243953

0.034067443

0.042072255

0.042072255

0.059994966

0.067027879

0.075684161
Genes in network

7

3

3

4

4

3

5

3

4

4

4

3

3

4
Genes in genome

14

12

11

66

90

35

283

43

145

161

161

61

65

199

Table (6): The gene co-expressed, shared domain and interaction with SCL25A15 gene network:

\begin{tabular}{cccl}
\hline Gene 1 & Gene 2 & Weight & Network group \\
\hline$G 6 P C$ & $A R G 1$ & 0.013130839 & Co-expression \\
$C T H$ & $S L C 25 A 15$ & 0.026114333 & Co-expression \\
$A R G 1$ & $H A L$ & 0.015584574 & Co-expression \\
$B A A T$ & $H A L$ & 0.010479293 & Co-expression \\
$B A A T$ & $A R G 1$ & 0.010356814 & Co-expression \\
$C 4 B P B$ & $B A A T$ & 0.009120464 & Co-expression \\
$M T H F S$ & $S L C 25 A 15$ & 0.025209978 & Co-expression \\
$M T H F S$ & $C T H$ & 0.023388306 & Co-expression \\
$M B L 2$ & $H A L$ & 0.012773992 & Co-expression \\
$M B L 2$ & $H G D$ & 0.00896276 & Co-expression \\
$C 5$ & $H A L$ & 0.011430247 & Co-expression \\
$C 5$ & $A R G 1$ & 0.01082647 & Co-expression \\
$C 5$ & $B A A T$ & 0.00760778 & Co-expression \\
$C 5$ & $C 4 B P B$ & 0.009530854 & Co-expression
\end{tabular}




\begin{tabular}{|c|c|c|c|}
\hline$C 5$ & $H G D$ & 0.007940412 & Co-expression \\
\hline$C 5$ & $M B L 2$ & 0.009273721 & Co-expression \\
\hline G6PC & $H A L$ & 0.011071949 & Co-expression \\
\hline G6PC & $B A A T$ & 0.007867279 & Co-expression \\
\hline G6PC & $C 4 B P B$ & 0.009524476 & Co-expression \\
\hline G6PC & $H G D$ & 0.007717506 & Co-expression \\
\hline$G 6 P C$ & $M B L 2$ & 0.009207115 & Co-expression \\
\hline G6PC & $C 5$ & 0.008035515 & Co-expression \\
\hline$L B P$ & $H A L$ & 0.009203292 & Co-expression \\
\hline$L B P$ & $A R G 1$ & 0.009164802 & Co-expression \\
\hline$L B P$ & $B A A T$ & 0.00658665 & Co-expression \\
\hline$L B P$ & $H G D$ & 0.006415015 & Co-expression \\
\hline$L B P$ & $M B L 2$ & 0.007615251 & Co-expression \\
\hline$L B P$ & $C 5$ & 0.006644955 & Co-expression \\
\hline$L B P$ & G6PC & 0.006901765 & Co-expression \\
\hline SLC17A2 & $H A L$ & 0.010264788 & Co-expression \\
\hline SLC17A2 & $B A A T$ & 0.007278958 & Co-expression \\
\hline SLC17A2 & $C 4 B P B$ & 0.009022474 & Co-expression \\
\hline SLC17A2 & $H G D$ & 0.007203176 & Co-expression \\
\hline SLC17A2 & $M B L 2$ & 0.008483202 & Co-expression \\
\hline SLC17A2 & $C 5$ & 0.007472467 & Co-expression \\
\hline SLC17A2 & G6PC & 0.007659466 & Co-expression \\
\hline SLC17A2 & $L B P$ & 0.006347752 & Co-expression \\
\hline ORM1 & $H A L$ & 0.009871504 & Co-expression \\
\hline ORMI & $A R G 1$ & 0.009783008 & Co-expression \\
\hline ORM1 & $B A A T$ & 0.007071007 & Co-expression \\
\hline ORM1 & $C 4 B P B$ & 0.008490616 & Co-expression \\
\hline ORM1 & $M B L 2$ & 0.008121029 & Co-expression \\
\hline ORMI & $C 5$ & 0.007148504 & Co-expression \\
\hline ORMI & G6PC & 0.007398048 & Co-expression \\
\hline ORMI & $L B P$ & 0.006209759 & Co-expression \\
\hline ORMI & SLC17A2 & 0.006826424 & Co-expression \\
\hline PREP & SLC25A15 & 0.010442503 & Co-expression \\
\hline SERPINA7 & $M B L 2$ & 0.013247599 & Co-expression \\
\hline CPS1 & $H A L$ & 0.01234286 & Co-expression \\
\hline$A R G 1$ & SLC25A15 & 0.005247516 & Co-expression \\
\hline$A R G 1$ & CTH & 0.005402988 & Co-expression \\
\hline$B A A T$ & SLC25A15 & 0.00938927 & Co-expression \\
\hline$B A A T$ & $H A L$ & 0.008351558 & Co-expression \\
\hline$B A A T$ & $A R G 1$ & 0.004348387 & Co-expression \\
\hline$C 4 B P B$ & SLC25A15 & 0.009231251 & Co-expression \\
\hline
\end{tabular}




\begin{tabular}{|c|c|c|c|}
\hline$C 4 B P B$ & $H A L$ & 0.00814306 & Co-expression \\
\hline$C 4 B P B$ & $B A A T$ & 0.007613069 & Co-expression \\
\hline$H G D$ & $H A L$ & 0.013029266 & Co-expression \\
\hline$H G D$ & $A R G 1$ & 0.006771524 & Co-expression \\
\hline$H G D$ & $B A A T$ & 0.012214398 & Co-expression \\
\hline$H G D$ & $C 4 B P B$ & 0.011807794 & Co-expression \\
\hline$M B L 2$ & SLC25A15 & 0.008736178 & Co-expression \\
\hline$M B L 2$ & $H A L$ & 0.007751754 & Co-expression \\
\hline$M B L 2$ & $B A A T$ & 0.007231998 & Co-expression \\
\hline$M B L 2$ & $C 4 B P B$ & 0.00703362 & Co-expression \\
\hline$M B L 2$ & $H G D$ & 0.011195325 & Co-expression \\
\hline$C 5$ & SLC25A15 & 0.006322735 & Co-expression \\
\hline$C 5$ & $H A L$ & 0.005552603 & Co-expression \\
\hline$C 5$ & $A R G 1$ & 0.002911721 & Co-expression \\
\hline$C 5$ & $B A A T$ & 0.005179715 & Co-expression \\
\hline$C 5$ & $C 4 B P B$ & 0.005058356 & Co-expression \\
\hline$C 5$ & $H G D$ & 0.008068559 & Co-expression \\
\hline$C 5$ & $M B L 2$ & 0.004826631 & Co-expression \\
\hline G6PC & $C T H$ & 0.006566833 & Co-expression \\
\hline G6PC & CPS1 & 0.007564845 & Co-expression \\
\hline G6PC & $A R G 1$ & 0.002987566 & Co-expression \\
\hline DIOI & $A R G 1$ & 0.003676503 & Co-expression \\
\hline DIOI & $C 5$ & 0.004387379 & Co-expression \\
\hline DIOI & $G 6 P C$ & 0.004473042 & Co-expression \\
\hline$L B P$ & $B A A T$ & 0.009965218 & Co-expression \\
\hline$L B P$ & $C 4 B P B$ & 0.009667832 & Co-expression \\
\hline$L B P$ & $M B L 2$ & 0.009191572 & Co-expression \\
\hline$L B P$ & $C 5$ & 0.006629785 & Co-expression \\
\hline SLC17A2 & SLC25A15 & 0.007915142 & Co-expression \\
\hline SLC17A2 & $H A L$ & 0.007040063 & Co-expression \\
\hline SLC17A2 & CPS1 & 0.009285137 & Co-expression \\
\hline SLC17A2 & $B A A T$ & 0.006526508 & Co-expression \\
\hline SLC17A2 & $C 4 B P B$ & 0.006351683 & Co-expression \\
\hline SLC17A2 & $H G D$ & 0.010178178 & Co-expression \\
\hline SLC17A2 & $M B L 2$ & 0.006092981 & Co-expression \\
\hline SLC17A2 & $C 5$ & 0.004395986 & Co-expression \\
\hline SLC17A2 & DIOI & 0.005542256 & Co-expression \\
\hline SLC17A2 & $L B P$ & 0.008360203 & Co-expression \\
\hline ORM1 & SLC25A15 & 0.009182534 & Co-expression \\
\hline ORMI & $C 5$ & 0.005041816 & Co-expression \\
\hline ORMI & $G 6 P C$ & 0.005162204 & Co-expression \\
\hline
\end{tabular}




\begin{tabular}{|c|c|c|c|}
\hline SERPINA7 & SLC25A15 & 0.007883273 & Co-expression \\
\hline SERPINA7 & $H A L$ & 0.006906412 & Co-expression \\
\hline SERPINA7 & CPS1 & 0.009286882 & Co-expression \\
\hline SERPINA7 & $A R G 1$ & 0.003641196 & Co-expression \\
\hline SERPINA7 & $C 4 B P B$ & 0.006316919 & Co-expression \\
\hline SERPINA7 & $M B L 2$ & 0.005971783 & Co-expression \\
\hline SERPINA7 & $C 5$ & 0.00436016 & Co-expression \\
\hline SERPINA7 & DIOI & 0.005475692 & Co-expression \\
\hline SERPINA7 & SLC17A2 & 0.005462843 & Co-expression \\
\hline SERPINA7 & ORM1 & 0.006366141 & Co-expression \\
\hline CYP3A5 & $C 4 B P B$ & 0.009888263 & Co-expression \\
\hline CYP3A5 & $M B L 2$ & 0.009454532 & Co-expression \\
\hline CYP3A5 & $C 5$ & 0.006786631 & Co-expression \\
\hline CYP $3 A 5$ & SLC17A2 & 0.008575552 & Co-expression \\
\hline CYP3A5 & ORMI & 0.009884344 & Co-expression \\
\hline CYP3A5 & SERPINA7 & 0.008449458 & Co-expression \\
\hline$C T H$ & SLC25A15 & 0.017681984 & Co-expression \\
\hline$H A L$ & SLC25A15 & 0.011282469 & Co-expression \\
\hline$H A L$ & CTH & 0.018665954 & Co-expression \\
\hline CPS1 & SLC25A15 & 0.014783018 & Co-expression \\
\hline CPS1 & CTH & 0.021131555 & Co-expression \\
\hline CPS1 & $H A L$ & 0.01605092 & Co-expression \\
\hline$A R G 1$ & SLC25A15 & 0.01022149 & Co-expression \\
\hline$A R G 1$ & CTH & 0.014448987 & Co-expression \\
\hline$A R G 1$ & CPS1 & 0.014791401 & Co-expression \\
\hline$B A A T$ & SLC25A15 & 0.007653142 & Co-expression \\
\hline$B A A T$ & CPS1 & 0.011089044 & Co-expression \\
\hline$B A A T$ & $A R G 1$ & 0.009111863 & Co-expression \\
\hline$C 4 B P B$ & $C T H$ & 0.007979206 & Co-expression \\
\hline$H G D$ & SLC25A15 & 0.009968358 & Co-expression \\
\hline$H G D$ & CTH & 0.011085113 & Co-expression \\
\hline$H G D$ & $H A L$ & 0.008449321 & Co-expression \\
\hline$H G D$ & CPS1 & 0.012882358 & Co-expression \\
\hline$H G D$ & $A R G 1$ & 0.009243058 & Co-expression \\
\hline$H G D$ & $B A A T$ & 0.007479178 & Co-expression \\
\hline MTHFS & $C 4 B P B$ & 0.009367498 & Co-expression \\
\hline$C 5$ & $H A L$ & 0.007322867 & Co-expression \\
\hline$C 5$ & $M B L 2$ & 0.010361436 & Co-expression \\
\hline$G 6 P C$ & SLC25A15 & 0.016148888 & Co-expression \\
\hline$G 6 P C$ & $C T H$ & 0.022236109 & Co-expression \\
\hline$G 6 P C$ & $H A L$ & 0.01545969 & Co-expression \\
\hline
\end{tabular}




\begin{tabular}{|c|c|c|c|}
\hline G6PC & CPS1 & 0.01768735 & Co-expression \\
\hline G6PC & $A R G 1$ & 0.012720793 & Co-expression \\
\hline G6PC & $B A A T$ & 0.009469871 & Co-expression \\
\hline G6PC & $H G D$ & 0.011001362 & Co-expression \\
\hline$L B P$ & $C 4 B P B$ & 0.003940297 & Co-expression \\
\hline$L E P R$ & SLC25A15 & 0.015385044 & Co-expression \\
\hline$L E P R$ & $H A L$ & 0.018157363 & Co-expression \\
\hline$L E P R$ & CPS1 & 0.021792563 & Co-expression \\
\hline$L E P R$ & $A R G 1$ & 0.012319317 & Co-expression \\
\hline$L E P R$ & $H G D$ & 0.012408955 & Co-expression \\
\hline$L E P R$ & $G 6 P C$ & 0.026779622 & Co-expression \\
\hline SLC17A2 & $C 4 B P B$ & 0.006601628 & Co-expression \\
\hline SLC17A2 & MTHFS & 0.011673527 & Co-expression \\
\hline SLC17A2 & $L B P$ & 0.004327369 & Co-expression \\
\hline ORM1 & $C 4 B P B$ & 0.008727606 & Co-expression \\
\hline ORMI & C5 & 0.007064989 & Co-expression \\
\hline PREP & SLC25A15 & 0.013786578 & Co-expression \\
\hline SERPINA7 & $C 5$ & 0.005918165 & Co-expression \\
\hline SERPINA7 & ORM1 & 0.010403195 & Co-expression \\
\hline$H G D$ & CPS1 & 0.020025367 & Co-expression \\
\hline DIOI & $M B L 2$ & 0.014330983 & Co-expression \\
\hline$L B P$ & G6PC & 0.0091696 & Co-expression \\
\hline CPS1 & SLC25A15 & 0.010167049 & Co-expression \\
\hline $\mathrm{CTH}$ & SLC25A15 & 0.022405656 & Co-expression \\
\hline$H A L$ & SLC25A15 & 0.024649095 & Co-expression \\
\hline CPS1 & SLC25A15 & 0.011441315 & Co-expression \\
\hline CPS1 & CTH & 0.011585185 & Co-expression \\
\hline CPS1 & $H A L$ & 0.012319576 & Co-expression \\
\hline$A R G 1$ & SLC25A15 & 0.020499837 & Co-expression \\
\hline$B A A T$ & SLC25A15 & 0.01333323 & Co-expression \\
\hline$B A A T$ & CTH & 0.01358016 & Co-expression \\
\hline$B A A T$ & CPS1 & 0.007241853 & Co-expression \\
\hline$B A A T$ & $A R G 1$ & 0.011780513 & Co-expression \\
\hline$C 4 B P B$ & SLC25A15 & 0.010655208 & Co-expression \\
\hline$C 4 B P B$ & CTH & 0.010559047 & Co-expression \\
\hline$C 4 B P B$ & $H A L$ & 0.011841011 & Co-expression \\
\hline$C 4 B P B$ & CPS1 & 0.006141116 & Co-expression \\
\hline$H G D$ & CPS1 & 0.009511196 & Co-expression \\
\hline$H G D$ & $B A A T$ & 0.011432248 & Co-expression \\
\hline$M B L 2$ & SLC25A15 & 0.017125212 & Co-expression \\
\hline$M B L 2$ & CTH & 0.016398663 & Co-expression \\
\hline
\end{tabular}




\begin{tabular}{|c|c|c|c|}
\hline$M B L 2$ & $H A L$ & 0.018915584 & Co-expression \\
\hline$M B L 2$ & CPS1 & 0.009279709 & Co-expression \\
\hline$M B L 2$ & $C 4 B P B$ & 0.008994715 & Co-expression \\
\hline$C 5$ & SLC25A15 & 0.008921804 & Co-expression \\
\hline$C 5$ & CTH & 0.008959468 & Co-expression \\
\hline$C 5$ & $H A L$ & 0.009587029 & Co-expression \\
\hline$C 5$ & CPS1 & 0.005092065 & Co-expression \\
\hline$C 5$ & $B A A T$ & 0.005769644 & Co-expression \\
\hline$C 5$ & $C 4 B P B$ & 0.004815416 & Co-expression \\
\hline$C 5$ & $H G D$ & 0.007569547 & Co-expression \\
\hline$C 5$ & $M B L 2$ & 0.007248661 & Co-expression \\
\hline G6PC & $C T H$ & 0.021676406 & Co-expression \\
\hline G6PC & CPS1 & 0.010997106 & Co-expression \\
\hline G6PC & $B A A T$ & 0.012542194 & Co-expression \\
\hline G6PC & $C 4 B P B$ & 0.009974403 & Co-expression \\
\hline G6PC & $H G D$ & 0.017824262 & Co-expression \\
\hline G6PC & $C 5$ & 0.00849682 & Co-expression \\
\hline$L B P$ & SLC25A15 & 0.018759409 & Co-expression \\
\hline$L B P$ & $H A L$ & 0.02087546 & Co-expression \\
\hline$L B P$ & CPS1 & 0.009439292 & Co-expression \\
\hline$L B P$ & $A R G 1$ & 0.018111857 & Co-expression \\
\hline$L B P$ & $B A A T$ & 0.010925728 & Co-expression \\
\hline$L B P$ & $C 4 B P B$ & 0.009051763 & Co-expression \\
\hline$L B P$ & $M B L 2$ & 0.014943773 & Co-expression \\
\hline$L B P$ & $C 5$ & 0.007415157 & Co-expression \\
\hline$L E P R$ & SLC25A15 & 0.025149433 & Co-expression \\
\hline$L E P R$ & $C T H$ & 0.02639034 & Co-expression \\
\hline$L E P R$ & CPS1 & 0.012923609 & Co-expression \\
\hline$L E P R$ & $B A A T$ & 0.015909543 & Co-expression \\
\hline$L E P R$ & $C 5$ & 0.010308038 & Co-expression \\
\hline$L E P R$ & $L B P$ & 0.020950127 & Co-expression \\
\hline SLC17A2 & $C 4 B P B$ & 0.005922639 & Co-expression \\
\hline SLC17A2 & $C 5$ & 0.00483539 & Co-expression \\
\hline ORM1 & SLC25A15 & 0.012226423 & Co-expression \\
\hline ORMI & CTH & 0.012432948 & Co-expression \\
\hline ORMI & $H A L$ & 0.013200064 & Co-expression \\
\hline ORMI & CPS1 & 0.006929017 & Co-expression \\
\hline ORMI & $B A A T$ & 0.007673539 & Co-expression \\
\hline ORMI & $C 4 B P B$ & 0.00653899 & Co-expression \\
\hline ORMI & $H G D$ & 0.010120259 & Co-expression \\
\hline ORMI & $M B L 2$ & 0.009900892 & Co-expression \\
\hline
\end{tabular}




\begin{tabular}{|c|c|c|c|}
\hline ORMI & $C 5$ & 0.005402325 & Co-expression \\
\hline ORMI & $G 6 P C$ & 0.011815394 & Co-expression \\
\hline ORMI & $L B P$ & 0.010073603 & Co-expression \\
\hline ORMI & $L E P R$ & 0.013810683 & Co-expression \\
\hline SERPINA7 & SLC25A15 & 0.012462362 & Co-expression \\
\hline SERPINA7 & $H A L$ & 0.01432913 & Co-expression \\
\hline SERPINA7 & CPS1 & 0.00686181 & Co-expression \\
\hline SERPINA7 & $A R G 1$ & 0.01146561 & Co-expression \\
\hline SERPINA7 & $C 4 B P B$ & 0.00677565 & Co-expression \\
\hline SERPINA7 & $M B L 2$ & 0.010809767 & Co-expression \\
\hline SERPINA7 & $C 5$ & 0.005448063 & Co-expression \\
\hline SERPINA7 & $L B P$ & 0.011217129 & Co-expression \\
\hline SERPINA7 & SLC17A2 & 0.006874819 & Co-expression \\
\hline CYP $3 A 5$ & CPS1 & 0.01207218 & Co-expression \\
\hline CYP3A5 & $C 4 B P B$ & 0.011685826 & Co-expression \\
\hline CYP3A5 & $C 5$ & 0.009578024 & Co-expression \\
\hline CYP3A5 & SLC17A2 & 0.011991991 & Co-expression \\
\hline CYP3A5 & ORM1 & 0.012806383 & Co-expression \\
\hline SLC17A2 & $G 6 P C$ & 0.014184606 & Co-expression \\
\hline SERPINA7 & $C 5$ & 0.019346774 & Co-expression \\
\hline CYP3A5 & SERPINA7 & 0.010969018 & Co-expression \\
\hline$H G D$ & SLC25A15 & 0.006551247 & Co-expression \\
\hline PREP & SLC25A15 & 0.012394655 & Co-expression \\
\hline SERPINA7 & G6PC & 0.017327776 & Co-expression \\
\hline CPS1 & $H A L$ & 0.00694992 & Co-expression \\
\hline$A R G 1$ & $\mathrm{CTH}$ & 0.006446471 & Co-expression \\
\hline$A R G 1$ & $H A L$ & 0.010185538 & Co-expression \\
\hline$A R G 1$ & CPS1 & 0.006081839 & Co-expression \\
\hline$B A A T$ & $A R G 1$ & 0.01191919 & Co-expression \\
\hline$C 4 B P B$ & CPS1 & 0.00363932 & Co-expression \\
\hline$H G D$ & CPS1 & 0.008691518 & Co-expression \\
\hline$H G D$ & $A R G 1$ & 0.010913373 & Co-expression \\
\hline MTHFS & $H A L$ & 0.007126623 & Co-expression \\
\hline$C 5$ & $C 4 B P B$ & 0.004553121 & Co-expression \\
\hline$L B P$ & $A R G 1$ & 0.006003315 & Co-expression \\
\hline$L B P$ & $C 4 B P B$ & 0.00376871 & Co-expression \\
\hline$L B P$ & $C 5$ & 0.005731973 & Co-expression \\
\hline$L E P R$ & CPS1 & 0.010181712 & Co-expression \\
\hline$L E P R$ & $A R G 1$ & 0.012883171 & Co-expression \\
\hline$L E P R$ & $H G D$ & 0.019583575 & Co-expression \\
\hline SERPINA7 & $C 4 B P B$ & 0.006879414 & Co-expression \\
\hline
\end{tabular}




\begin{tabular}{|c|c|c|c|}
\hline SERPINA7 & $C 5$ & 0.009770601 & Co-expression \\
\hline SERPINA7 & DIOI & 0.018938221 & Co-expression \\
\hline SERPINA7 & SLC17A2 & 0.021433447 & Co-expression \\
\hline DIOI & $C 4 B P B$ & 0.01167786 & Co-expression \\
\hline DIOI & $C T H$ & 0.004786647 & Co-expression \\
\hline DIOI & $M B L 2$ & 0.007709226 & Co-expression \\
\hline DIOI & C5 & 0.006562013 & Co-expression \\
\hline$H A L$ & $\mathrm{CTH}$ & 0.00904644 & Co-localization \\
\hline$A R G 1$ & $\mathrm{CTH}$ & 0.007396141 & Co-localization \\
\hline$A R G 1$ & $H A L$ & 0.011341748 & Co-localization \\
\hline$C 4 B P B$ & $\mathrm{CTH}$ & 0.00882447 & Co-localization \\
\hline$C 4 B P B$ & $H A L$ & 0.009825678 & Co-localization \\
\hline$M B L 2$ & $A R G 1$ & 0.011475822 & Co-localization \\
\hline$C 5$ & $C T H$ & 0.009882752 & Co-localization \\
\hline$C 5$ & $H A L$ & 0.012378897 & Co-localization \\
\hline$C 5$ & $A R G 1$ & 0.011783864 & Co-localization \\
\hline$C 5$ & $M B L 2$ & 0.015168445 & Co-localization \\
\hline$G 6 P C$ & $C T H$ & 0.006479372 & Co-localization \\
\hline$G 6 P C$ & $H A L$ & 0.007433198 & Co-localization \\
\hline$G 6 P C$ & $A R G 1$ & 0.006618402 & Co-localization \\
\hline$G 6 P C$ & $C 4 B P B$ & 0.008764179 & Co-localization \\
\hline$G 6 P C$ & $H G D$ & 0.011694877 & Co-localization \\
\hline SERPINA7 & $\mathrm{CTH}$ & 0.007116361 & Co-localization \\
\hline SERPINA7 & $H A L$ & 0.008624428 & Co-localization \\
\hline SERPINA7 & $A R G 1$ & 0.007838565 & Co-localization \\
\hline SERPINA7 & $C 4 B P B$ & 0.009594075 & Co-localization \\
\hline SERPINA7 & $M B L 2$ & 0.011912366 & Co-localization \\
\hline SERPINA7 & $C 5$ & 0.012075914 & Co-localization \\
\hline SERPINA7 & $G 6 P C$ & 0.007159275 & Co-localization \\
\hline$H A L$ & SLC25A15 & 0.014272561 & Co-localization \\
\hline CPS1 & SLC25A15 & 0.013714614 & Co-localization \\
\hline$A R G 1$ & SLC25A15 & 0.01310207 & Co-localization \\
\hline$A R G 1$ & CPS1 & 0.00861983 & Co-localization \\
\hline$B A A T$ & SLC25A15 & 0.013363565 & Co-localization \\
\hline$B A A T$ & CPS1 & 0.008450216 & Co-localization \\
\hline$B A A T$ & $A R G 1$ & 0.008598406 & Co-localization \\
\hline$H G D$ & SLC25A15 & 0.022047134 & Co-localization \\
\hline$H G D$ & $H A L$ & 0.010812771 & Co-localization \\
\hline MTHFS & SLC25A15 & 0.008358937 & Co-localization \\
\hline MTHFS & $H A L$ & 0.004322442 & Co-localization \\
\hline MTHFS & $C 4 B P B$ & 0.009622298 & Co-localization \\
\hline
\end{tabular}




\begin{tabular}{|c|c|c|c|}
\hline MTHFS & $H G D$ & 0.007224194 & Co-localization \\
\hline$A D C K 2$ & SLC25A15 & 0.026127955 & Co-localization \\
\hline$A D C K 2$ & $B A A T$ & 0.010211586 & Co-localization \\
\hline$A D C K 2$ & MTHFS & 0.00685143 & Co-localization \\
\hline$M B L 2$ & SLC25A15 & 0.011515018 & Co-localization \\
\hline$M B L 2$ & $C 4 B P B$ & 0.012219642 & Co-localization \\
\hline$M B L 2$ & MTHFS & 0.003996356 & Co-localization \\
\hline$M B L 2$ & $A D C K 2$ & 0.009659463 & Co-localization \\
\hline$C 5$ & SLC25A15 & 0.01863199 & Co-localization \\
\hline$C 5$ & $H A L$ & 0.009531116 & Co-localization \\
\hline$C 5$ & CPS1 & 0.010474401 & Co-localization \\
\hline$C 5$ & $A R G 1$ & 0.010341191 & Co-localization \\
\hline$C 5$ & $B A A T$ & 0.010462028 & Co-localization \\
\hline G6PC & SLC25A15 & 0.016094055 & Co-localization \\
\hline G6PC & $H A L$ & 0.008763849 & Co-localization \\
\hline G6PC & CPS1 & 0.009082945 & Co-localizatio \\
\hline G6PC & $B A A T$ & 0.00880316 & Co-localization \\
\hline G6PC & $H G D$ & 0.014180019 & Co-localization \\
\hline$G 6 P C$ & MTHFS & 0.005369584 & Co-localizatio \\
\hline$G 6 P C$ & $M B L 2$ & 0.007431859 & Co-localizatio \\
\hline$G 6 P C$ & $C 5$ & 0.011984772 & Co-localization \\
\hline DIOI & SLC25A15 & 0.025056243 & Co-localization \\
\hline DIOI & $H G D$ & 0.017162617 & Co-localization \\
\hline$L B P$ & SLC25A15 & 0.014860843 & Co-localization \\
\hline$L B P$ & $H A L$ & 0.008506832 & Co-localization \\
\hline$L B P$ & $B A A T$ & 0.006969759 & Co-localization \\
\hline$L B P$ & $H G D$ & 0.011018015 & Co-localization \\
\hline$L B P$ & MTHFS & 0.004518892 & Co-localization \\
\hline$L B P$ & $M B L 2$ & 0.006218935 & Co-localization \\
\hline$L B P$ & $C 5$ & 0.010104029 & Co-localization \\
\hline$L B P$ & G6PC & 0.009210799 & Co-localization \\
\hline SLC17A2 & SLC25A15 & 0.018329779 & Co-localization \\
\hline SLC17A2 & $H A L$ & 0.009681415 & Co-localization \\
\hline SLC17A2 & $B A A T$ & 0.009294453 & Co-localization \\
\hline SLC17A2 & $H G D$ & 0.013592821 & Co-localization \\
\hline SLC17A2 & MTHFS & 0.006027925 & Co-localization \\
\hline SLC 17A2 & $A D C K 2$ & 0.015540332 & Co-localization \\
\hline SLC17A2 & $M B L 2$ & 0.008607964 & Co-localization \\
\hline SLC17A2 & $G 6 P C$ & 0.011782652 & Co-localization \\
\hline SLC17A2 & $L B P$ & 0.010201342 & Co-localization \\
\hline ORM1 & SLC25A15 & 0.009743731 & Co-localization \\
\hline
\end{tabular}




\begin{tabular}{|c|c|c|c|}
\hline ORMI & $H A L$ & 0.005098609 & Co-localization \\
\hline ORMI & CPS1 & 0.005120896 & Co-localization \\
\hline ORMI & $A R G 1$ & 0.004895931 & Co-localization \\
\hline ORMI & $B A A T$ & 0.004949312 & Co-localization \\
\hline ORMI & $C 4 B P B$ & 0.009870878 & Co-localization \\
\hline ORM1 & $H G D$ & 0.007168311 & Co-localization \\
\hline ORMI & MTHFS & 0.003196832 & Co-localization \\
\hline ORM1 & $A D C K 2$ & 0.007305417 & Co-localization \\
\hline ORMI & $M B L 2$ & 0.004552235 & Co-localization \\
\hline ORMI & $C 5$ & 0.006662394 & Co-localization \\
\hline ORMI & G6PC & 0.006180191 & Co-localization \\
\hline ORMI & $L B P$ & 0.005398762 & Co-localization \\
\hline ORM1 & $S L C 17 A 2$ & 0.006999721 & Co-localization \\
\hline SERPINA7 & SLC25A15 & 0.010623649 & Co-localization \\
\hline SERPINA7 & CPS1 & 0.006563142 & Co-localization \\
\hline SERPINA7 & $A R G 1$ & 0.006524967 & Co-localization \\
\hline SERPINA7 & $B A A T$ & 0.006416609 & Co-localization \\
\hline SERPINA7 & $M B L 2$ & 0.005163964 & Co-localization \\
\hline SERPINA7 & $C 5$ & 0.00801424 & Co-localization \\
\hline SERPINA7 & G6PC & 0.007043994 & Co-localization \\
\hline SERPINA7 & $L B P$ & 0.005624746 & Co-localization \\
\hline SERPINA7 & SLC17A2 & 0.007645443 & Co-localization \\
\hline SERPINA7 & ORM1 & 0.004100054 & Co-localization \\
\hline CYP3A5 & SLC25A15 & 0.021976126 & Co-localization \\
\hline CYP3A5 & $H A L$ & 0.012220652 & Co-localization \\
\hline CYP3A5 & CPS1 & 0.011446337 & Co-localization \\
\hline CYP3A5 & $B A A T$ & 0.011028161 & Co-localization \\
\hline CYP3A5 & MTHFS & 0.006549534 & Co-localization \\
\hline CYP3A5 & C5 & 0.015651088 & Co-localization \\
\hline CYP3A5 & G6PC & 0.01435386 & Co-localization \\
\hline CYP $3 A 5$ & $L B P$ & 0.012599541 & Co-localization \\
\hline CYP3A5 & SLC17A2 & 0.014993709 & Co-localization \\
\hline CYP3A5 & ORM1 & 0.008000645 & Co-localization \\
\hline CYP3A5 & SERPINA7 & 0.008649936 & Co-localization \\
\hline PREP & CTH & 0.047972582 & Physical Interactions \\
\hline$C 4 B P B$ & SLC25A15 & 0.15971017 & Physical Interactions \\
\hline
\end{tabular}




\section{D analysis of SLC25A15 gene by UCSF Chimera and Project HOPE:}

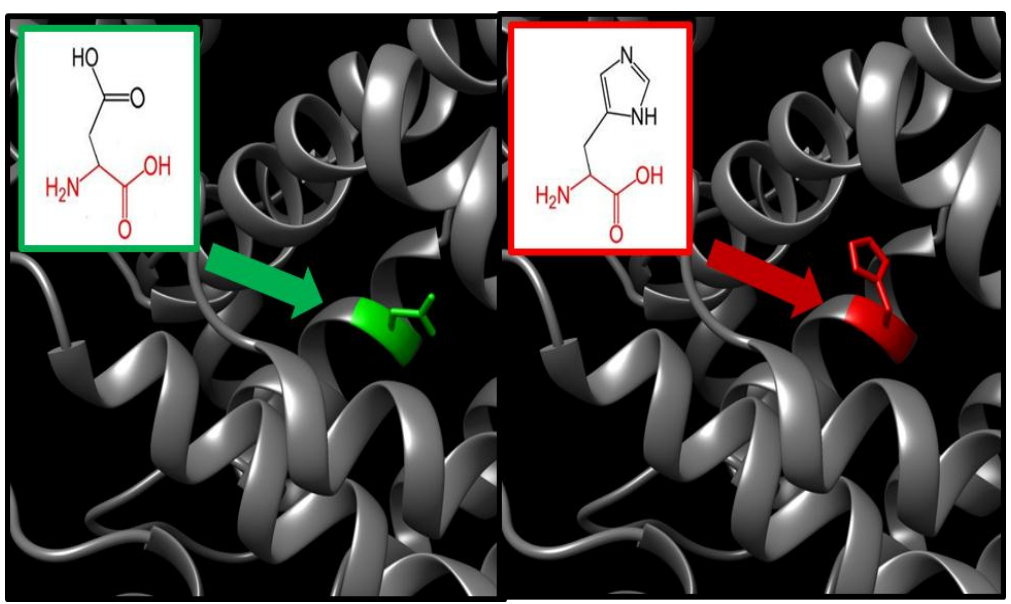

Figure 2: (G31H) the amino acid Aspartic Acid (green) changes to Histidine (red) at position 31.

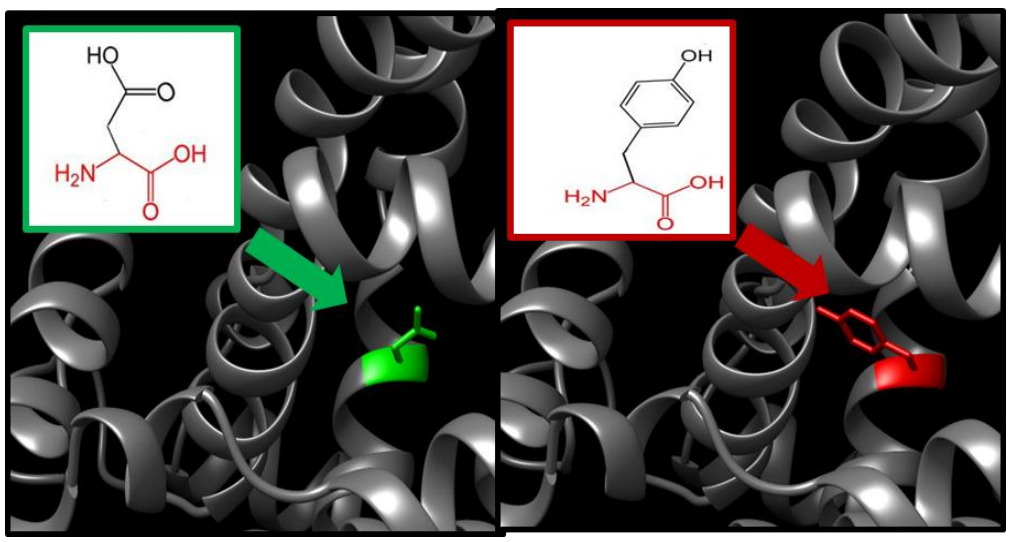

Figure 3: (D31Y) the amino acid Aspartic Acid (green) changes to Tyrosine (red) at position 31.

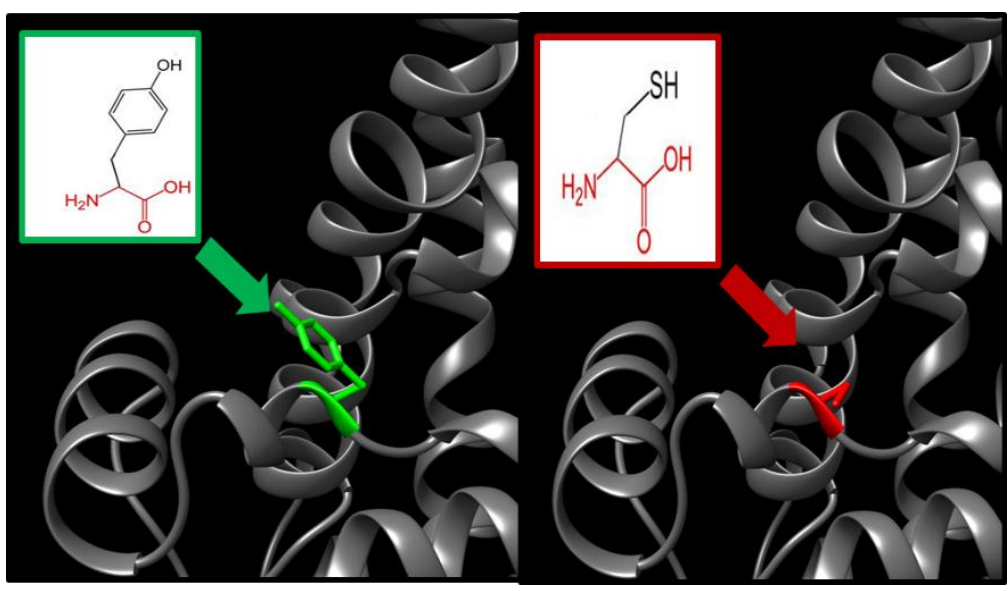

Figure 4: (Y64C) the amino acid Tyrosine (green) changes into a Cysteine (red) at position 64 


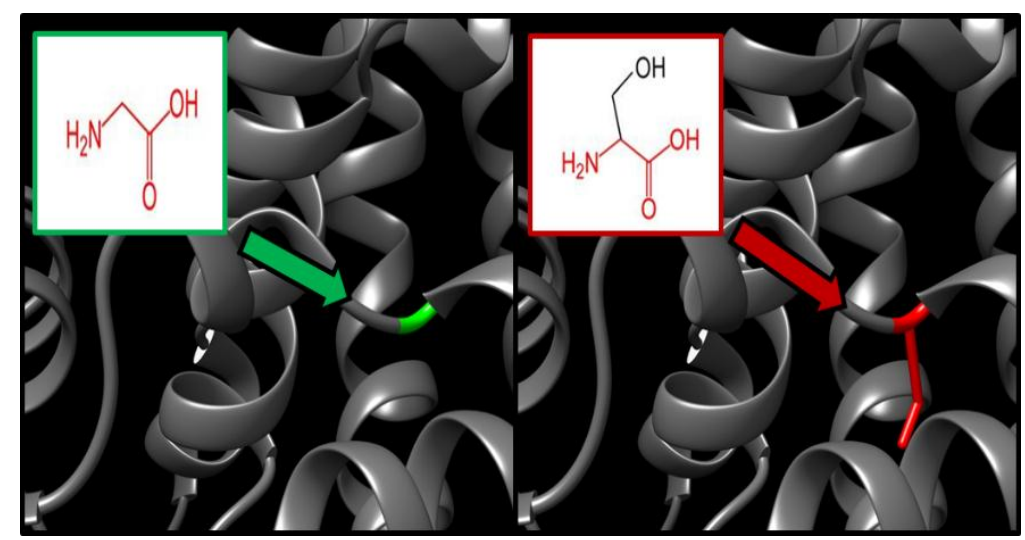

Figure 5: (G66S) the amino acid Glycine (green) changes into a Serine (red) at position 66.

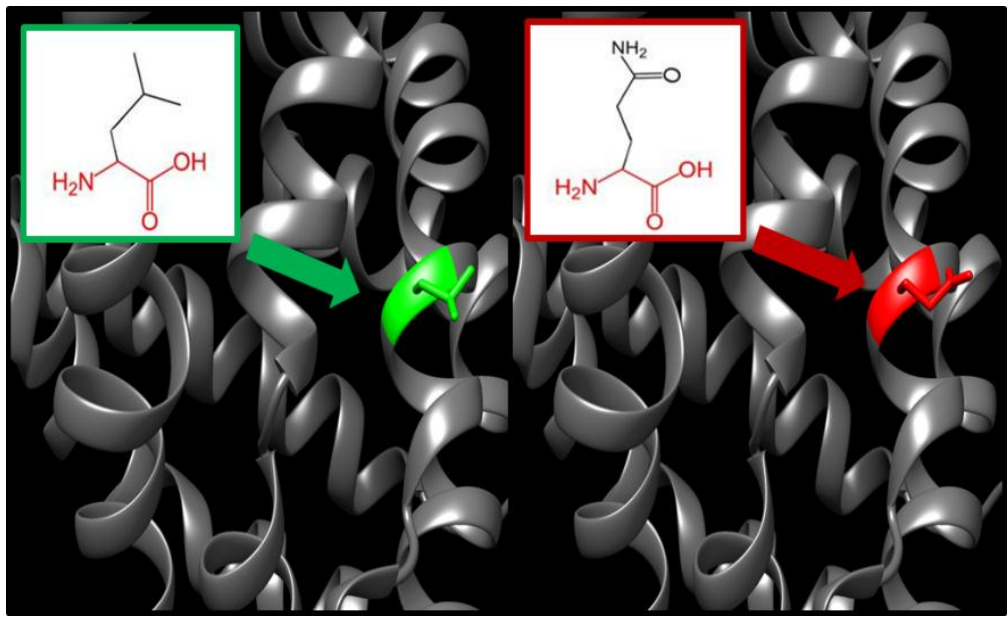

Figure 6: (L71Q) the amino acid Leucine (green) changes into a Glutamine (red) at position 71.

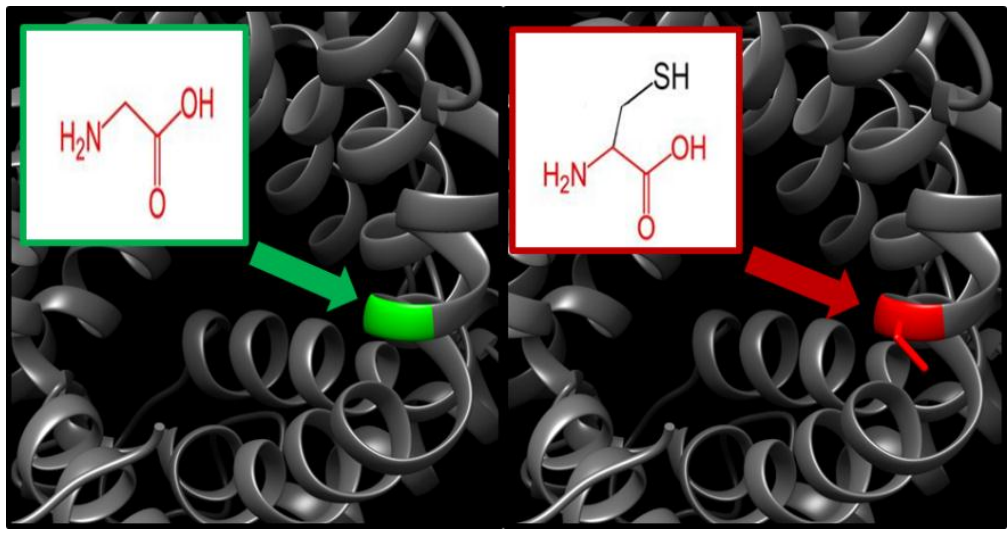

Figure 7: (G86C) the amino acid Glycine (green) changes into a Cysteine (red) at position 86. 


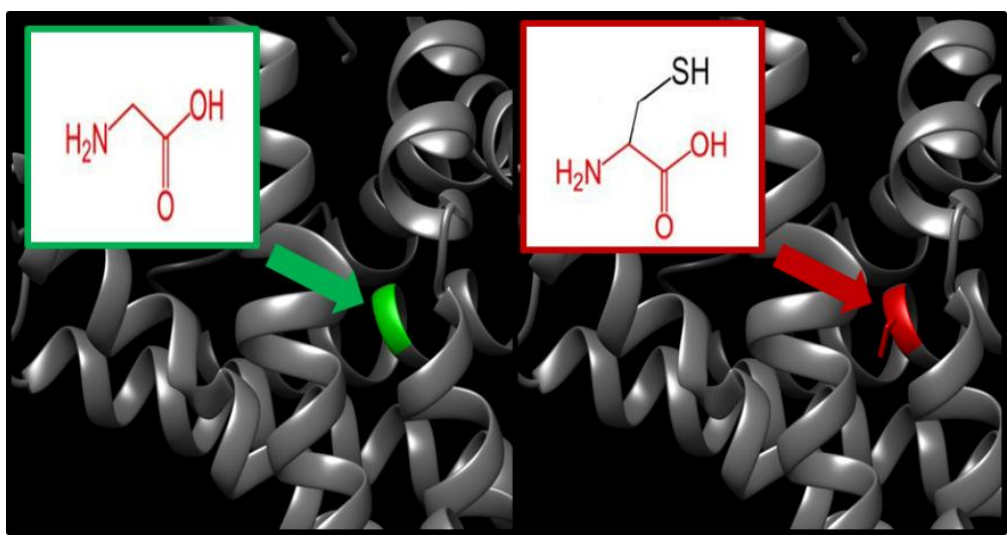

Figure 8: (G113C) the amino Acid Glycine (green) changes into a Cysteine (red) at position 113.

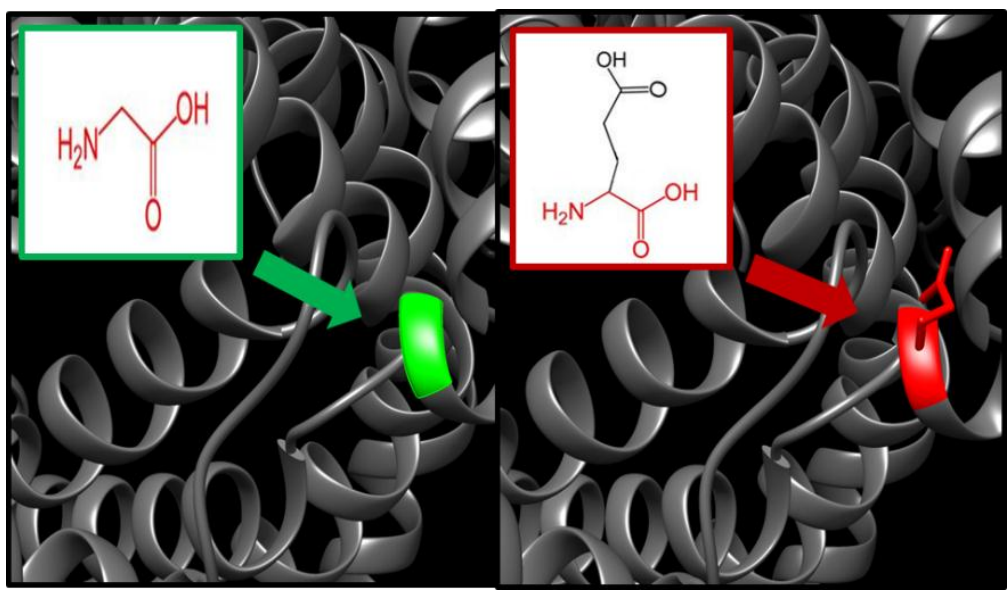

Figure 9: (G168E) the amino acid Glycine (green) changes into a Glutamic Acid (red) at position 168.

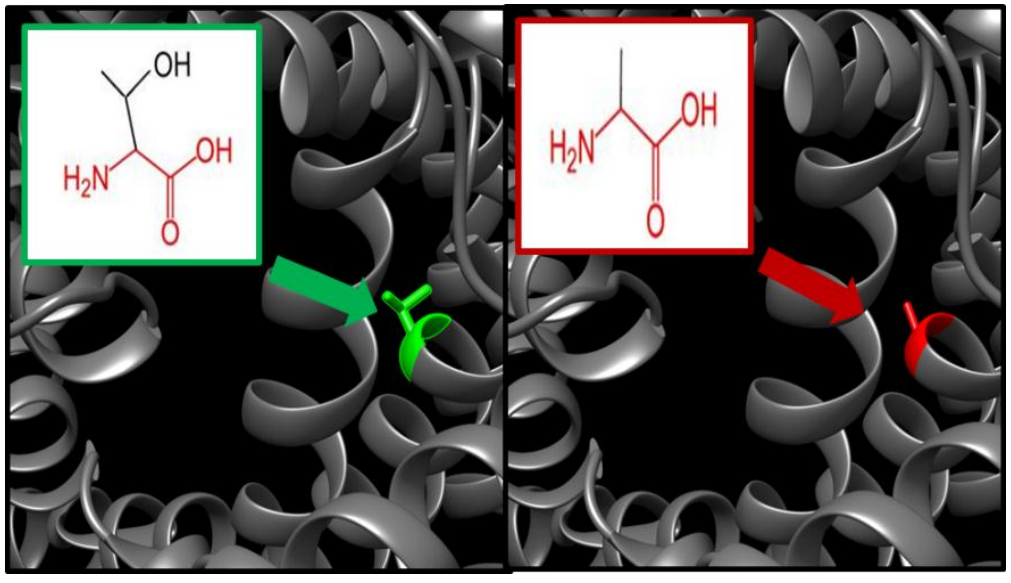

Figure 10: (T176A) the amino acid Threonine (green) changes into Alanine (red) at position 176. 


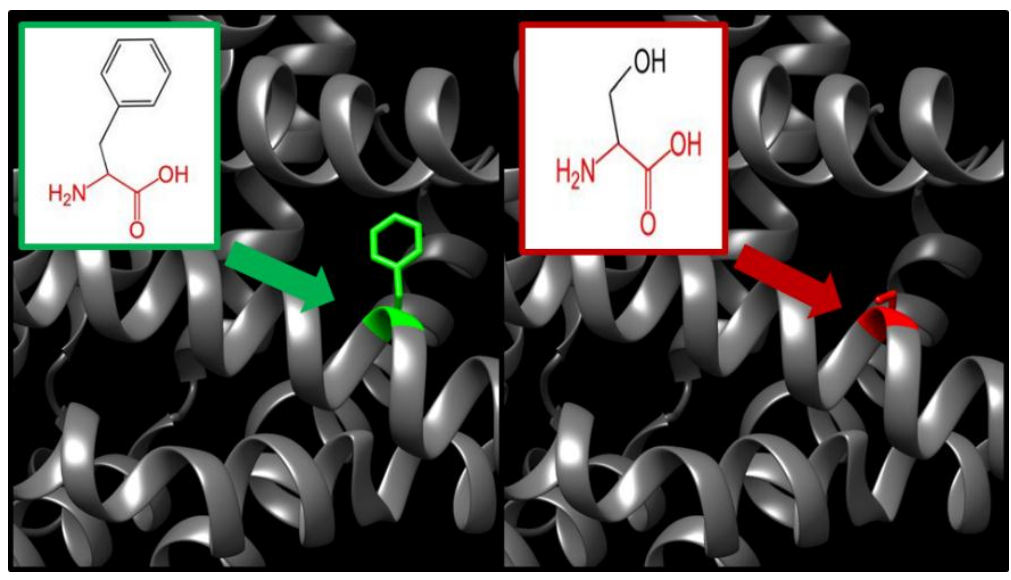

Figure 11: (F186S) the amino acid Phenylalanine (green) changes to Serine (red) at position 188.

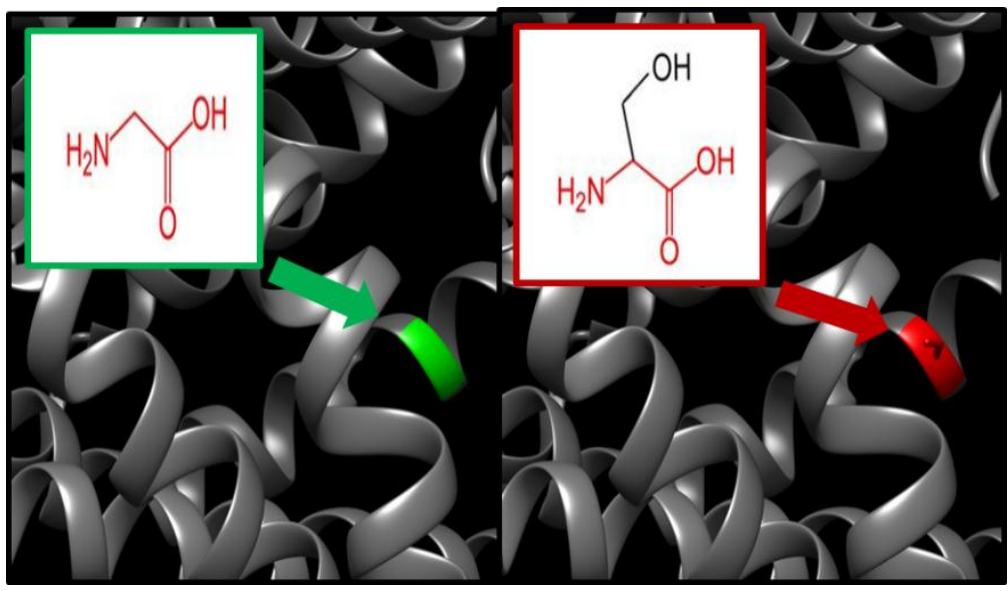

Figure 12: (G216S) the amino acid Glycine (green) changes to Serine 9 (red) at position 216.

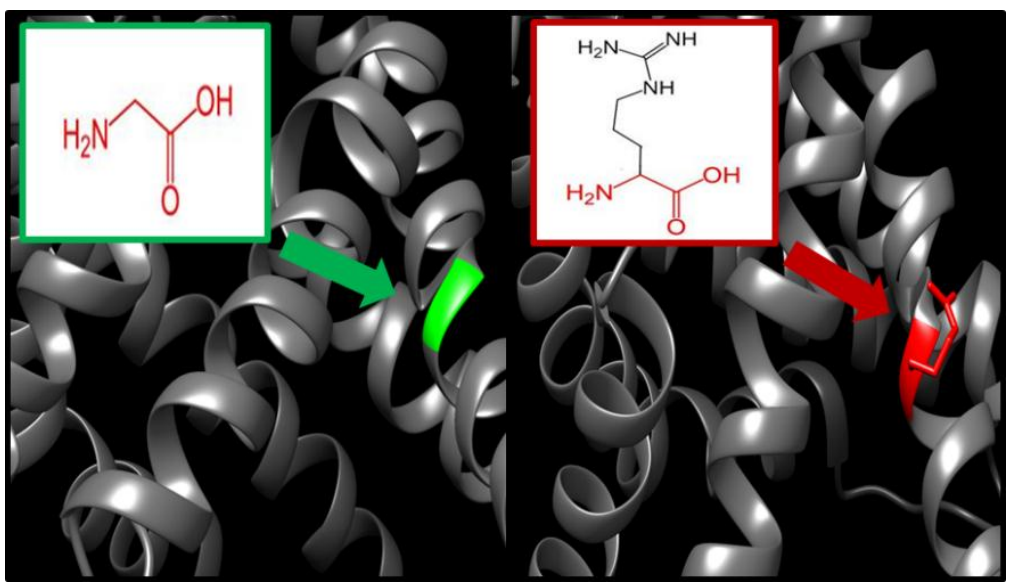

Figure 13: (G217R) the amino acid Glycine (green) change to Arginine (red) at position 217 


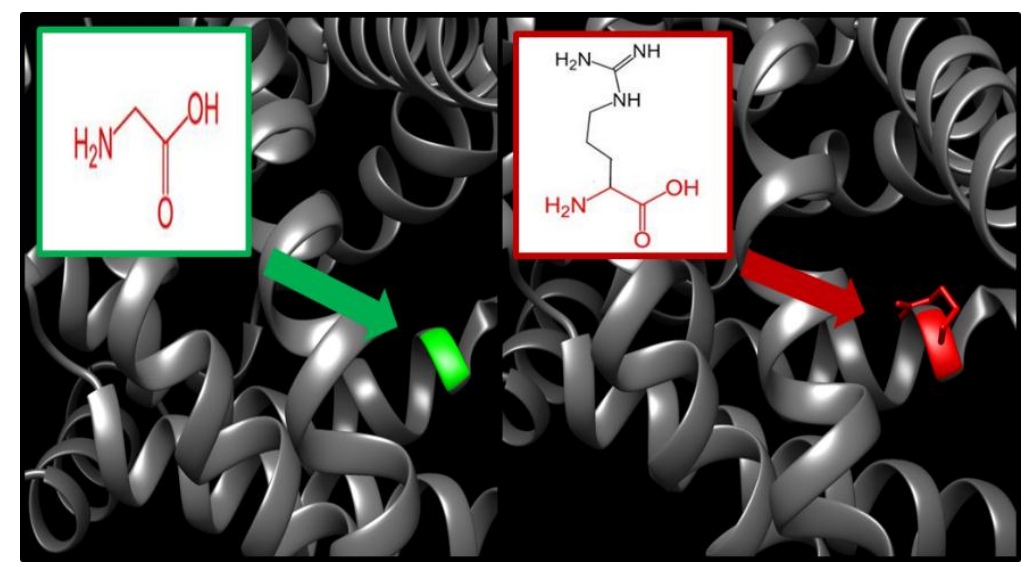

Figure 14: (G220R) the amino acid Glycine (green) change to Arginine (red) at position 220.

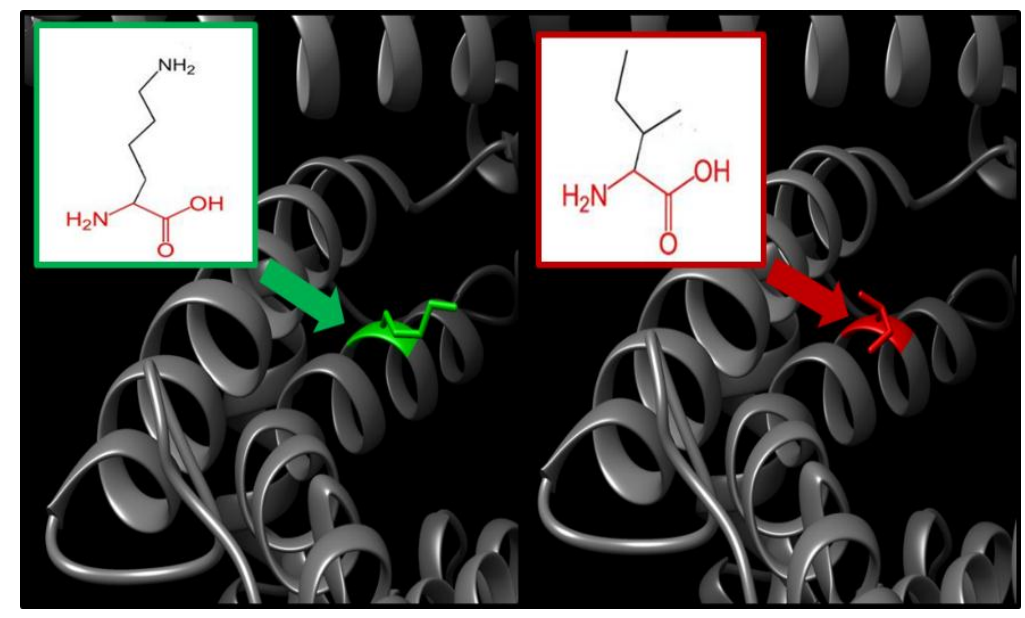

Figure 15: (K234I) the amino acid Lysine (green) changes to Isoleucine (red) at position 234.

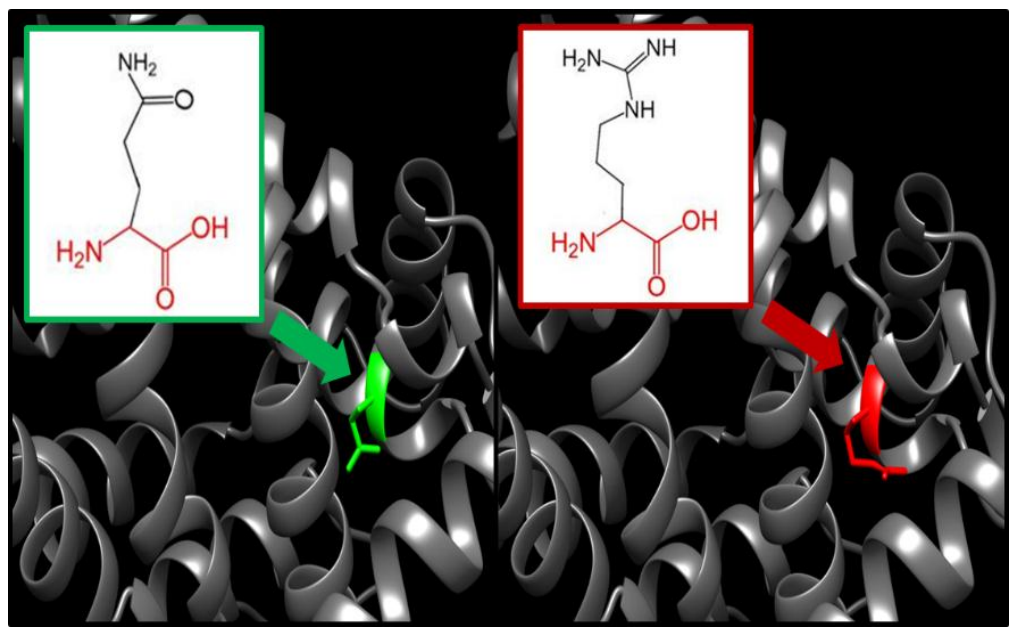

Figure 16: (Q238R) the amino acid Glutamine (green) changes to Arginine (red) at position 238. 


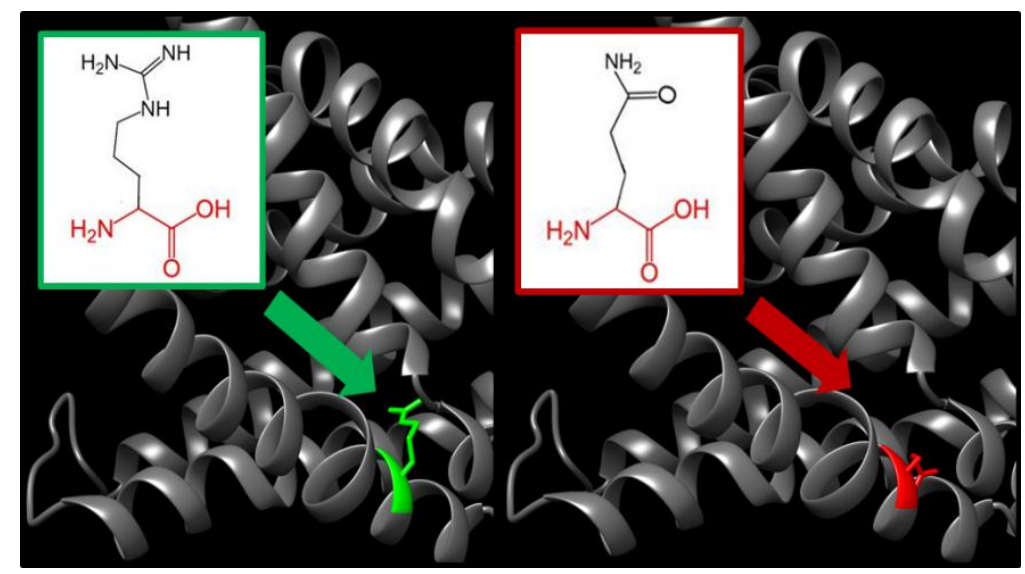

Figure 17: (R275Q) the amino acid Arginine (green) changes to Glutamine (red) at position 275.

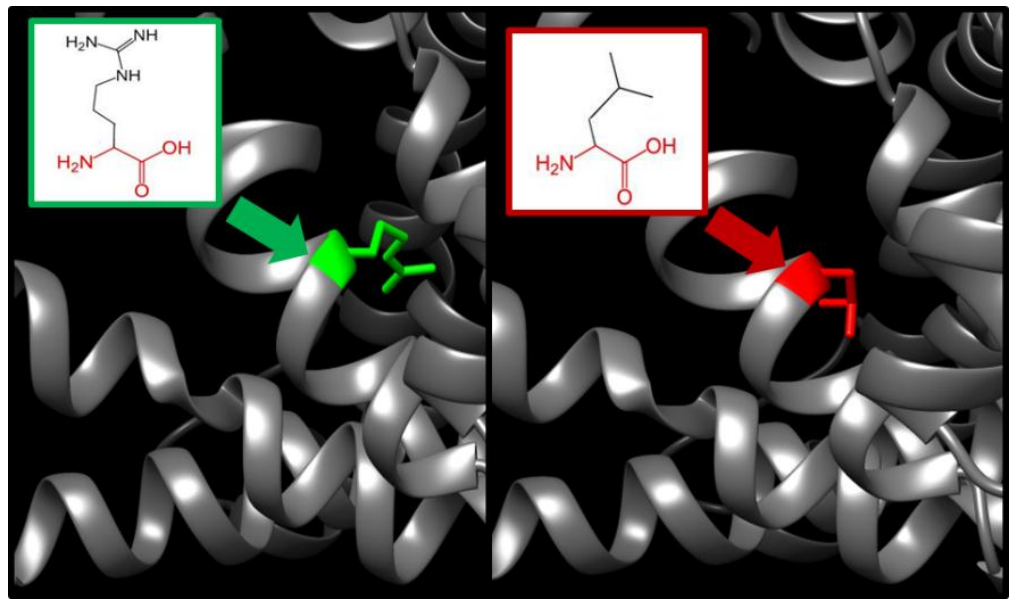

Figure 18: (R275L) the amino acid Arginine (green) change to Leucine (red) at position 275.

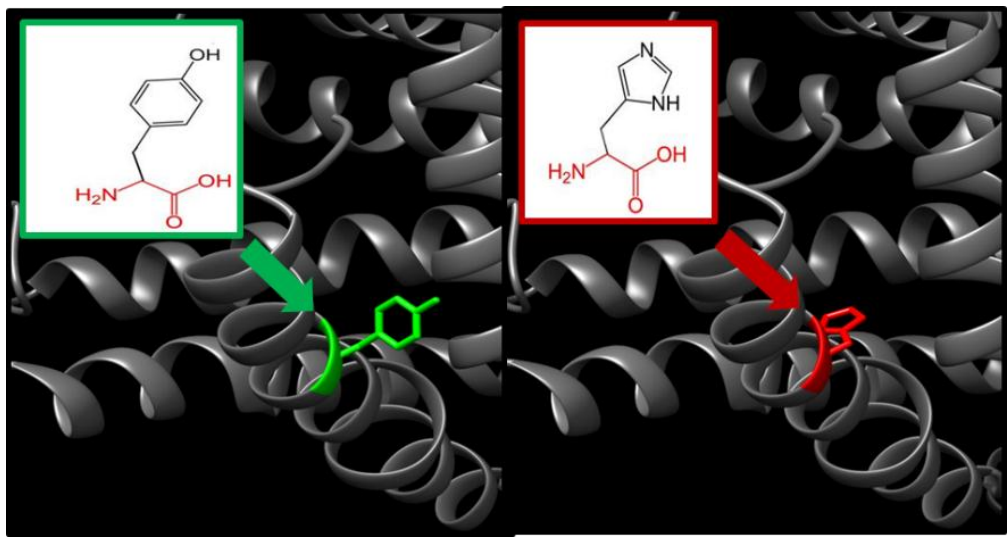

Figure 19: (Y287H) the amino acid Tyrosine (green) changes to Histidine (red) at position 287. 


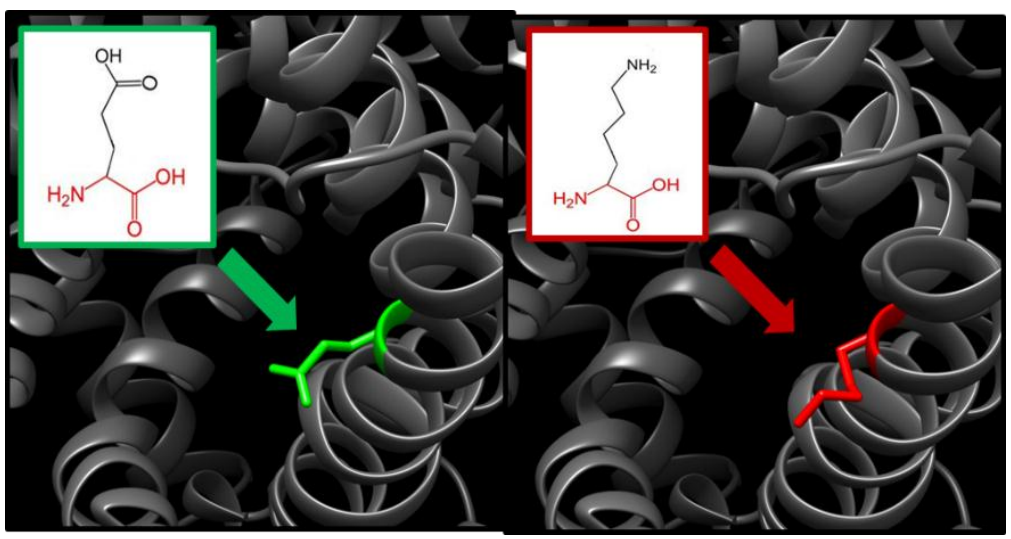

Figure 20: (E288K) the amino acid Glutamic Acid (green) changes to Lysine (red) at position 288.

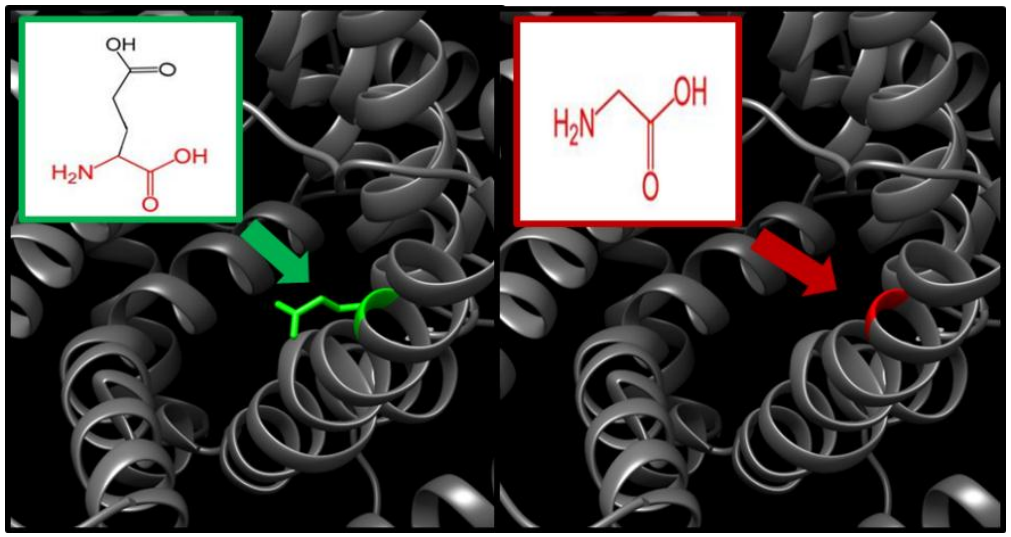

Figure 21: (E288G) the amino acid Glutamic Acid (green) changes into a Glycine (red) at position 288. 


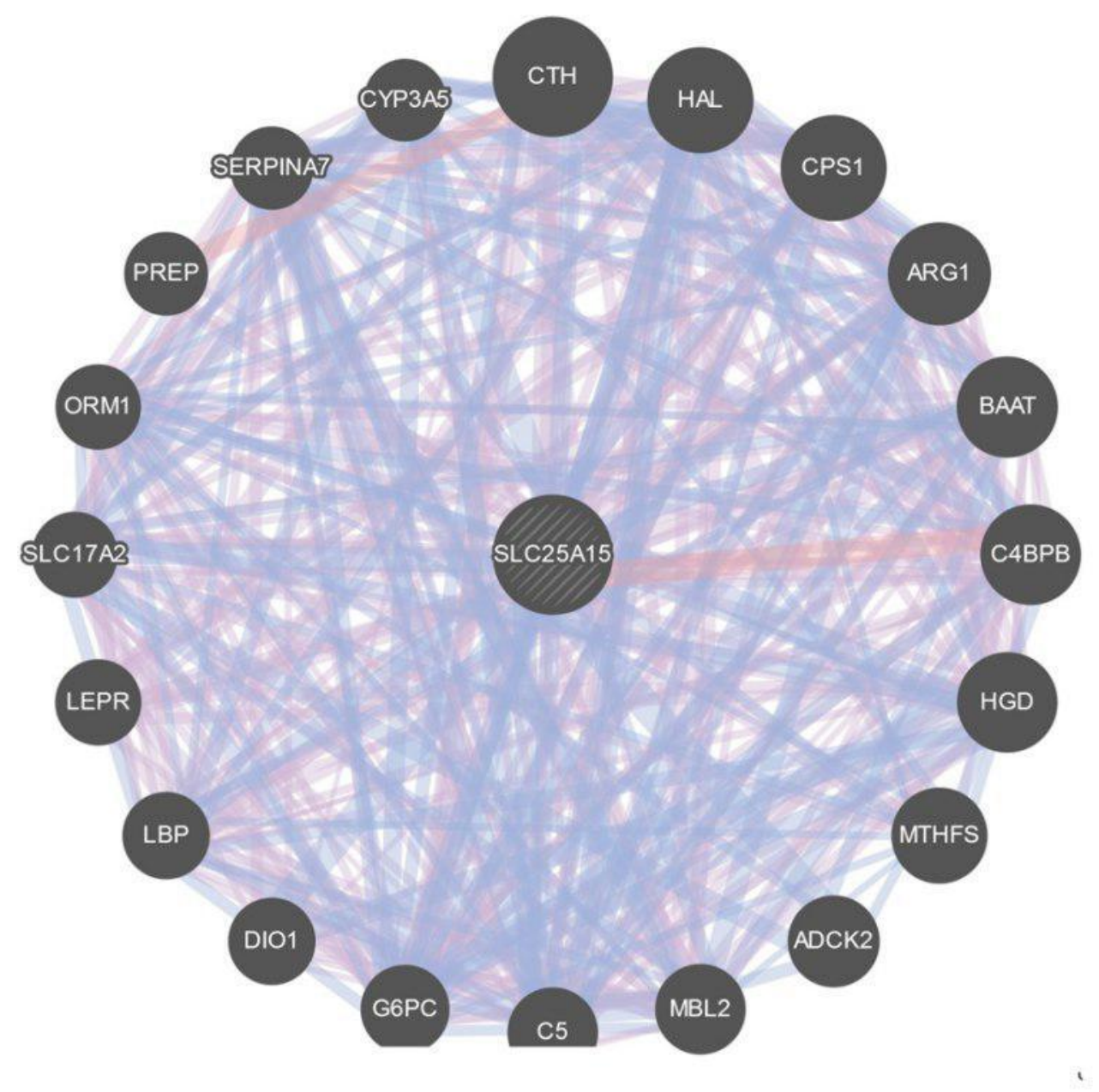

\section{Networks}

Physical Interactions

Co-expression

Predicted

Co-localization

Pathway

Genetic Interactions

Shared protein domains

Figure22: Interaction between $S L C 25 A 15$ and its related genes. 


\subsection{Discussion:}

Various bioinformatics tools were performed to identify the SNPs impact in the SLC25A15 protein. 20 mutations have been distinguished to affect the stability and function of SLC25A15, 12 out of 20 are considered as novel (D31H, D31Y, Y64C, G66S, G86C, G168E, T176A, F188S, K234I, Y287H, E288K and E288G). To ensure the pathological effect of the mutations at the molecular level, different parameters plus trustworthy information were used, and for more accuracy, numerous methods rather than one method (PolyPhen-2, PROVEAN, PHD-SNP, SNP\&GO, PANTHER, P-MUT, I-Mutant3.0, MUpro, Project HOPE, RaptorX, Chimera, and GeneMANIA were used (Figure1)

630 variants of the Homo Sapiens SLC25A15 gene which situated on the coding region were acquired from dbSNP/NCBI Database. To predict if these variants affect the function of the protein, phenotype and end by disease 220 missense mutations were submitted to SIFT, PolyPhen-2 and PROVEAN software respectively. 57 SNPs were predicted to be 'deleterious' by SIFT software, while by PolypPhen-2; 114 SNPs were predicted to be 'damaging', in which 79 were 'probably damaging' and 35 as 'possibly damaging ', and PROVEAN predicted 142 SNPs to be deleterious. The triple positive results of these software were 27 SNPs, and they are affecting the function of the protein (Table 1). These 27 pathogenic SNPs were submitted to PHD-SNP, SNP\&GO, PANTHER and P-MUT for further confirmation; 27 deleterious SNPs were predicted by PHD-SNP and SNP\&GO while PANTHER predict 25 variants and P-MUT predicted 21 deleterious SNPs (table 2 AND 3). The fourth positive results of these software were 20 pathogenic SNPs, were submitted to I-Mutant and MUpro servers in order to examine the effect of mutation on the stability of the protein. I-Mutant revealed all these 20 pathogenic SNPs decreased the stability of the protein except for 4 SNPs with I-Mutant (D31Y, G168E, G216S, and G217R), but one SNP with MUPRO (K234I) was predicted to increase the stability (Table 4).

Project HOPE reveled that some mutant residues (which are buried in the core of the protein) are larger than the wild-type residues, this makes the new residue in an improper position to make the same hydrogen bond as the original wild-type residue did, while the smaller sized mutants cannot fill the core of the protein. It also showed the mutation may change the charge of the residue and lead to repulsion of ligands or other residues with the same charge, there are 9 variants remain unchanged. In Project HOPE results, 12 variants with mutant residue are more hydrophobic than the wild-type residue, these hydrophobicity variations can affect the hydrophobic interactions with the membrane lipids, and it identified 10 variants with transmembrane domain as well. The amino acid Glycine is the most flexible of all residues, this flexibility might be necessary for the protein's function. Thus mutation of this glycine can abolish this function. For visualization of the wild and mutant types of amino acids in SLC25A15, Chimera software was performed (Figures 2-21)

GeneMania disclosed that SLC25A15 functions in the amide biosynthetic process, nitrogen cycle metabolic process, urea metabolic process, cellular amino acid catabolic process, inflammatory response, complement activation, cellular modified amino acid metabolic process, carboxylic acid catabolic process, organic acid catabolic process, protein activation cascade. Disruption of this function give rise to cytoplasmic ornithine aggregation, citrulline synthesis reduction, impaired ammonia detoxication and increased carbamoyl phosphate(1). The genes co-expressed with; share similar protein domain, or participate to achieve similar function were illustrated by GeneMANIA and shown in figure (22) Tables (5, and 6). 
This study is the first in silico analysis to determine the impact of SNPs on the coding region of the SLC25A15, other studies relied on molecular work investigated, specifically the effect of mutations on SLC25A15 that specified and associated with phenotype of HHH syndrome (26). Also, detailed information on HHH was carried out by analysis of SLC25A15 gene with computational modeling NMR, in order to study and construct the 3D structure and mutated mitochondrial ornithine transporter 1 , and also to provide molecular docking to identify substrate binding site followed by dynamic simulation to optimize these model (51).

20 pathogenic SNPs effect on the SLC25A15 protein function and structure were reveled in this study. 12 of these 20 missense SNPs are proposedly to be considered as novel mutations, while; L71Q, G113C, F188S, G216S, G220R, Q238R, R275Q, and R275L are not originals, due to their previous mentions in studies $(1,11,12,26,52,53)$.

NCBI and this study both acknowledge that L71Q, G113S, G220R, and R275Q SNPs to be pathogenic, in addition to, this study also found further 16 other pathogenic SNPs. However underneath NCBI pathogenic results; G27R was to be positive, while in this study G27R resulted as not pathogenic SNP.

Overexpression of SLC25A15 may enhance the melanoma proliferation, which might affect the melanoma prognosis and future orientation for melanoma therapy (53).

The diagnosis of HHH associates with clinical manifestation of the syndrome, while treatment may improve with protein free diet and administration of arginine, however liver transplant may still be required (54). This new study, it is not only costless, but can rapidly identify the pathogenic SNPs, specifically targeting mutant SLC25A15 and may aid in biomarker diagnosis of the disease thus allowing safe novel gene therapy to be performed, from the alternative liver transplant.

\section{Conclusions:}

In this study 20 pathogenic SNPs (D31Y, Y64C, G66S, L71Q, G86C, G113C, G168E, T176A, F188S, G216S, G217R, G220R, K234I, Q238R, R275Q, R275L, Y287H, E288K, and E288G) were found in SLC25A15 gene through a combination of different in silico bioinformatics tools. Their impact on SLC25A15 gene functions and structure were also analyzed, thus providing knowledge as a starting point for innovation of new, useful SNP markers for medical testing and a safer medication to treat the most common demoralizing disorders of SLC25A15 in urea cycle. 


\section{References:}

1. Tessa A, Fiermonte G, Dionisi-Vici C, Paradies E, Baumgartner MR, Chien YH, et al. Identification of novel mutations in the SLC25A15 gene in hyperornithinemia-hyperammonemiahomocitrullinuria (HHH) syndrome: A clinical, molecular, and functional study. 2009;30(5):7418.

2. Summar ML, Mew NAJPC. Inborn errors of metabolism with hyperammonemia: Urea cycle defects and related disorders. 2018;65(2):231-46.

3. Mitchell S, Ellingson C, Coyne T, Hall L, Neill M, Christian N, et al. Genetic variation in the urea cycle: a model resource for investigating key candidate genes for common diseases. 2009;30(1):56-60.

4. $\quad$ Stone WL, Jaishankar GBJSTI. Urea Cycle Disorders. 2018.

5. Mew NA, Simpson KL, Gropman AL, Lanpher BC, Chapman KA, Summar ML. Urea cycle disorders overview. GeneReviews ${ }^{\circledR}$ [Internet]: University of Washington, Seattle; 2017.

6. Debray F-G, Lambert M, Lemieux B, Soucy J, Drouin R, Fenyves D, et al. Phenotypic variability among patients with hyperornithinaemia-hyperammonaemia-homocitrullinuria syndrome homozygous for the delF188 mutation in SLC25A15. 2008;45(11):759-64.

7. Tunalı NE, Marobbio CM, Tiryakioğlu NO, Punzi G, Saygılı SK, Önal H, et al. A novel mutation in the SLC25A15 gene in a Turkish patient with HHH syndrome: functional analysis of the mutant protein. 2014;112(1):25-9.

8. Marobbio CM, Punzi G, Pierri CL, Palmieri L, Calvello R, Panaro MA, et al. Pathogenic potential of SLC25A15 mutations assessed by transport assays and complementation of Saccharomyces cerevisiae ORT1 null mutant. 2015;115(1):27-32.

9. $\quad$ PaTHWay MJIMDiAACG. 20| HHH SYNDROME. 2016:137.

10. Filosto M, Alberici A, Tessa A, Padovani A, Santorelli FMJNS. Hyperornithinemiahyperammonemia-homocitrullinuria $(\mathrm{HHH})$ syndrome in adulthood: a rare recognizable condition. 2013;34(9):1699-701.

11. Tezcan K, Louie KT, Qu Y, Velasquez J, Zaldivar F, Rioseco-Camacho N, et al. Adultonset presentation of a hyperornithinemia-hyperammonemia-homocitrullinuria patient without prior history of neurological complications. JIMD Reports-Case and Research Reports, 2011/3: Springer; 2011. p. 97-102.

12. Silfverberg T, Sahlander F, Enlund M, Oscarson M, Hårdstedt MJJomcr. Late onset hyperornithinemia-hyperammonemia-homocitrullinuria syndrome-how web searching by the family solved unexplained unconsciousness: a case report. 2018;12(1):274.

13. Camacho J, Rioseco-Camacho N. Hyperornithinemia-Hyperammonemia-

Homocitrullinuria Syndrome. In: Adam MP, Ardinger HH, Pagon RA, Wallace SE, Bean LJH, Stephens K, et al., editors. GeneReviews((R)). Seattle (WA): University of Washington, Seattle

University of Washington, Seattle. GeneReviews is a registered trademark of the University of Washington, Seattle. All rights reserved.; 1993.

14. Filosto M, Alberici A, Tessa A, Padovani A, Santorelli FM. Hyperornithinemiahyperammonemia-homocitrullinuria $(\mathrm{HHH})$ syndrome in adulthood: a rare recognizable condition. Neurological sciences : official journal of the Italian Neurological Society and of the Italian Society of Clinical Neurophysiology. 2013;34(9):1699-701.

15. Palmieri FJJoimd. Mitochondrial transporters of the SLC25 family and associated diseases: a review. 2014;37(4):565-75.

16. Torisu H, Kira R, Kanazawa N, Takemoto M, Sanefuji M, Sakai Y, et al. A novel R275X mutation of the SLC25A15 gene in a Japanese patient with the HHH syndrome. 2006;28(5):3325.

17. Korman SH, Kanazawa N, Abu-Libdeh B, Gutman A, Tsujino SJJotns.

Hyperornithinemia, hyperammonemia, and homocitrullinuria syndrome with evidence of 
mitochondrial dysfunction due to a novel SLC25A15 (ORNT1) gene mutation in a Palestinian family. 2004;218(1-2):53-8.

18. Salvi S, Dionisi-Vici C, Bertini E, Verardo M, Santorelli FJHm. Seven novel mutations in the ORNT1 gene (SLC25A15) in patients with hyperornithinemia, hyperammonemia, and homocitrullinuria syndrome. 2001;18(5):460-.

19. Viegas CM, Tonin AM, Zanatta Â, Seminotti B, Busanello ENB, Fernandes CG, et al. Impairment of brain redox homeostasis caused by the major metabolites accumulating in hyperornithinemia-hyperammonemia-homocitrullinuria syndrome in vivo. 2012;27(4):521-30.

20. Jang KM, Hyun MC, Hwang SKJJotKCNS. A Novel SLC25A15 Mmutation Causing Hyperornithinemia-Hyperammonemia-Homocitrullinuria Syndrome. 2017;25(3):204-7.

21. Fiermonte G, Dolce V, David L, Santorelli FM, Dionisi-Vici C, Palmieri F, et al. The Mitochondrial Ornithine Transporter BACTERIAL EXPRESSION, RECONSTITUTION, FUNCTIONAL CHARACTERIZATION, AND TISSUE DISTRIBUTION OF TWO HUMAN ISOFORMS. 2003;278(35):32778-83.

22. Martinelli D, Diodato D, Ponzi E, Monne M, Boenzi S, Bertini E, et al. The hyperornithinemia-hyperammonemia-homocitrullinuria syndrome. Orphanet journal of rare diseases. 2015;10:29.

23. Lee HH, Poon KH, Lai CK, Au KM, Siu TS, Lai JP, et al. Hyperornithinaemiahyperammonaemia-homocitrullinuria syndrome: a treatable genetic liver disease warranting urgent diagnosis. Hong Kong medical journal = Xianggang yi xue za zhi. 2014;20(1):63-6.

24. Leonard J, Morris A, editors. Urea cycle disorders. Seminars in neonatology; 2002:

Elsevier.

25. Machado MC, da Silva FPJJoic. Hyperammonemia due to urea cycle disorders: a potentially fatal condition in the intensive care setting. 2014;2(1):22.

26. Martinelli D, Diodato D, Ponzi E, Monné M, Boenzi S, Bertini E, et al. The hyperornithinemia-hyperammonemia-homocitrullinuria syndrome. 2015;10(1):29.

27. Kingsmore SJPc. Comprehensive carrier screening and molecular diagnostic testing for recessive childhood diseases. 2012;4.

28. Sokoro AA, Lepage J, Antonishyn N, McDonald R, Rockman-Greenberg C, Irvine J, et al. Diagnosis and high incidence of hyperornithinemia-hyperammonemia-homocitrullinemia (HHH) syndrome in northern Saskatchewan. Journal of inherited metabolic disease. 2010;33 Suppl 3:S275-81.

29. Ceravolo F, Sestito S, Falvo F, Salpietro V, Polizzi A, Ruggieri M, et al. Neurological Involvement in Inherited Metabolic Diseases: An Overview. 2016;6(01):003-10.

30. Viecelli HM, Thöny BJJoPB. Challenges of experimental gene therapy for urea cycle disorders. 2014;4(01):065-73.

31. Tenenbaum JDJG, proteomics, bioinformatics. Translational bioinformatics: past, present, and future. 2016;14(1):31-41.

32. Samadian E, Gharaei R, Colagar AH, Sohrabi HJJoGE, Biotechnology. Computational study of putative functional variants in human kisspeptin. 2017;15(2):419-22.

33. Ng PC, Henikoff SJNar. SIFT: Predicting amino acid changes that affect protein function. 2003;31(13):3812-4.

34. Sim N-L, Kumar P, Hu J, Henikoff S, Schneider G, Ng PCJNar. SIFT web server: predicting effects of amino acid substitutions on proteins. 2012;40(W1):W452-W7.

35. Adzhubei I, Jordan DM, Sunyaev SRJCpihg. Predicting functional effect of human missense mutations using PolyPhen-2. 2013;76(1):7.20. 1-7.. 41.

36. Capriotti E, Altman RBJBb. Improving the prediction of disease-related variants using protein three-dimensional structure. 2011;12(4):S3.

37. Choi Y, Sims GE, Murphy S, Miller JR, Chan APJPo. Predicting the functional effect of amino acid substitutions and indels. 2012;7(10):e46688. 
38. Choi Y, Chan APJB. PROVEAN web server: a tool to predict the functional effect of amino acid substitutions and indels. 2015;31(16):2745-7.

39. Ou L, Przybilla MJ, Whitley CBJOjord. Phenotype prediction for mucopolysaccharidosis type I by in silico analysis. 2017;12(1):125.

40. Capriotti E, Calabrese R, Fariselli P, Martelli PL, Altman RB, Casadio RJBg. WSSNPs\&GO: a web server for predicting the deleterious effect of human protein variants using functional annotation. 2013;14(3):S6.

41. Calabrese R, Capriotti E, Fariselli P, Martelli PL, Casadio RJHm. Functional annotations improve the predictive score of human disease-related mutations in proteins. 2009;30(8):1237-44.

42. Ferrer-Costa C, Gelpí JL, Zamakola L, Parraga I, De La Cruz X, Orozco MJB. PMUT: a web-based tool for the annotation of pathological mutations on proteins. 2005;21(14):3176-8.

43. Cruz Xdl, Orozco M, Gelpí JL, editors. Web-based tool for the annotation of pathological variants on proteins: PMut 2017 update. Book of abstracts; 2017: Barcelona Supercomputing Center.

44. Capriotti E, Fariselli P, Casadio RJNar. I-Mutant2. 0: predicting stability changes upon mutation from the protein sequence or structure. 2005;33(suppl_2):W306-W10.

45. Cheng J, Randall A, Baldi PJPS, Function,, Bioinformatics. Prediction of protein stability changes for single-site mutations using support vector machines. 2006;62(4):1125-32.

46. Venselaar H, te Beek TA, Kuipers RK, Hekkelman ML, Vriend GJBb. Protein structure analysis of mutations causing inheritable diseases. An e-Science approach with life scientist friendly interfaces. 2010;11(1):548.

47. Wang S, Li W, Liu S, Xu JJNar. RaptorX-Property: a web server for protein structure property prediction. 2016;44(W1):W430-W5.

48. Pettersen EF, Goddard TD, Huang CC, Couch GS, Greenblatt DM, Meng EC, et al. UCSF Chimera - a visualization system for exploratory research and analysis. 2004;25(13):160512.

49. Franz M, Rodriguez H, Lopes C, Zuberi K, Montojo J, Bader GD, et al. GeneMANIA update 2018. 2018;46(W1):W60-W4.

50. Warde-Farley D, Donaldson SL, Comes O, Zuberi K, Badrawi R, Chao P, et al. The GeneMANIA prediction server: biological network integration for gene prioritization and predicting gene function. 2010;38(suppl_2):W214-W20.

51. Wang J-F, Chou K-CJPO. Insights into the mutation-induced HHH syndrome from modeling human mitochondrial ornithine transporter-1. 2012;7(1):e31048.

52. Shih VE, Laframboise R, Mandell R, Pichette JJPd. Neonatal form of the hyperornithinaemia, hyperammonaemia, and homocitrullinuria $(\mathrm{HHH})$ syndrome and prenatal diagnosis. 1992;12(9):717-23.

53. Fecarotta S, Parenti G, Vajro P, Zuppaldi A, Casa RD, Carbone M, et al. HHH syndrome (hyperornithinaemia, hyperammonaemia, homocitrullinuria), with fulminant hepatitis-like presentation. 2006;29(1):186-9.

54. Mhanni A, Chan A, Collison M, Seifert B, Lehotay D, Sokoro A, et al. Hyperornithinemia-hyperammonemia-homocitrullinuria syndrome $(\mathrm{HHH})$ presenting with acute fulminant hepatic failure. 2008;46(3):312-5. 